\title{
Cosmological singularities and 2-dimensional dilaton gravity
}

\author{
Ritabrata Bhattacharya, K. Narayan and Partha Paul \\ Chennai Mathematical Institute, \\ SIPCOT IT Park, Siruseri 603103, India \\ E-mail: ritabratab3@gmail.com, narayan@cmi.ac.in, \\ pl.partha13@gmail.com
}

ABstract: We study Big-Bang or -Crunch cosmological singularities in 2-dimensional dilaton-gravity-scalar theories, in general obtained by dimensional reduction of higher dimensional theories. The dilaton potential encodes information about the asymptotic data defining the theories, and encompasses various families such as flat space, AdS, conformally AdS as arising from nonconformal branes, and more general nonrelativistic theories. We find a kind of universal near singularity behaviour independent of the dilaton potential, giving universal interrelations between the exponents defining the time behaviour near the cosmological singularity. More detailed analysis using a scaling ansatz enables finding various classes of cosmological backgrounds, recovering known examples such as the AdS Kasner singularity as well finding as new ones. We give some comments on the dual field theory from this point of view.

KEYwords: 2D Gravity, Black Holes in String Theory, Gauge-gravity correspondence

ArXiv ePrint: 2006.09470 


\section{Contents}

1 Introduction 1

$2 \quad A d S / C F T$ and Big-Bang/Crunch singularities 2

3 Redux to 2d dilaton gravity: Big-Crunch attractors 3

4 Solving the 2-dim theory for Big-Crunches $\quad 7$

4.1 AdS and conformally AdS backgrounds 8

$\begin{array}{lll}4.2 & \text { Hyperscaling violating Lifshitz asymptotics } & 11\end{array}$

$\begin{array}{lll}4.3 & \text { JT gravity } & 15\end{array}$

$\begin{array}{lll}5 & \text { Some comments on the dual field theory } & 17\end{array}$

$\begin{array}{llr}6 & \text { Discussion } & 20\end{array}$

\section{Introduction}

It is a fond hope that string theory sheds light on Big Bang or Crunch singularities and the early universe: there is a rich history of previous investigations, e.g. [1]-[53] (see also e.g. $[54,55]$ for reviews). Some of these involve the worldsheet theory of strings propagating in the background of cosmological singularities, the intuition being that the extended nature of the string (its oscillatory modes and interactions thereof) smoothes out the near singularity region. Others involve holography [56]-[59], the hope being that the dual field theory is well-defined even if the bulk gravitational theory breaks down in the vicinity of the singularity. For instance, time-dependent deformations of $A d S / C F T$ were studied in [24]-[27]: the bulk develops a Big-Crunch singularity and breaks down while the dual field theory subjected to severe time-dependence (in some cases due to a severe time-varying gauge coupling) may be hoped to march on: we will discuss these more later. In many cases however, the analysis, while interesting and instructive, suggests a singular response in the field theory as well. Further insights on some of these were obtained in [39]-[42] in part via different holographic screens. It would be desirable to have more conclusive insights into possible resolutions of cosmological singularities in string theory.

In this paper, we study time-dependent backgrounds in 2-dimensional dilaton-gravitymatter theories, many of which are obtained by dimensional reduction from higher dimensional theories. The 2-dim theories here contain the 2-dim dilaton coupling to gravity as well as an extra scalar and a dilaton potential. Some of the cosmological singularities known in flat space and in the $A d S / C F T$ deformations mentioned above are encompassed in these 2dim theories: although the reduction ansatz precludes very anisotropic backgrounds, it still allows interesting structure. This recasting into 2-dimensions helps organize the equations 
of motion governing such Big-Bang or -Crunch singularities: we will often find it convenient to think in terms of a Crunch. In particular we find a kind of "universal near singularity" behaviour near the (spacelike) Big-Crunch, defined by a region where the fields are rapidly varying in time leading to large time derivatives that eventually diverge: in this region the precise form of the dilaton potential turns out to essentially disappear, thus becoming irrelevant. This suggests a scaling ansatz for the pertinent 2-dimensional fields, i.e. the metric function $e^{f}$, dilaton $X$ and scalar $e^{\Phi}$, which is of power-law form in both time $t$ and space $r$ variables. The universal near singularity behaviour above implies universal relations between the exponents governing the time behaviour near the singularity: in particular these relations are satisfied by the flat space Kasner singularity that is "mostly isotropic".

A more detailed analysis of the 2-dimensional equations using this scaling ansatz confirms this "cosmological attractor" type behaviour and also fixes the precise values of all the exponents. In particular this recovers the familiar flat space Kasner and AdS Kasner Big Bang singularities: as well as various familiar time-independent backgrounds such as $A d S$, conformally $A d S$ and nonrelativistic theories. Performing this analysis for more general dilaton potentials obtained by reductions of certain classes of nonrelativistic theories in fact reveals new families of time-dependent backgrounds with cosmological singularities and hyperscaling violating Lifshitz asymptotics far from the singular region. In JT gravity, this reveals a cosmological background exhibiting Crunch behaviour where the dilaton vanishes.

In section 2, we briefly review certain aspects of the investigations in [24-27] on $A d S / C F T$ and Big-Bang singularities. Section 3 describes the effective 2-dimensional dilaton-gravity-matter theories that encode such time-dependent backgrounds, in particular those arising as reductions from higher dimensions. Section 4 discusses more detailed analysis on solving the 2-dim theory for Big-Bang or -Crunch singularities, in various theories. Section 5 has some comments on the holographic duals in the AdS/CFT cosmological singularities and we conclude finally with a Discussion in section 6 .

\section{$2 \quad A d S / C F T$ and Big-Bang/Crunch singularities}

Time-dependent non-normalizable deformations of $A d S / C F T$ were studied in [24-27] towards gaining insights via gauge/gravity duality into cosmological (Big-Bang or -Crunch) singularities: some of these were further investigated in [39-42]. The bulk gravity theory exhibits a cosmological Big-Crunch (or -Bang) singularity and breaks down while the holographic dual field theory (in the $A d S_{5}$ case) subject to a severe time-dependent gauge coupling $g_{\mathrm{YM}}^{2}=e^{\Phi}$ may be hoped to provide insight into the dual dynamics: in this case the scalar $\Phi$ controls the gauge/string coupling. There is a large family of such backgrounds exhibiting cosmological singularities (we will discuss them further in section 5). For instance, we obtain AdS-Kasner theories as

$$
d s^{2}=\frac{R_{\mathrm{AdS}}^{2}}{r^{2}}\left(-d t^{2}+\sum_{i} t^{2 p_{i}} d x_{i}^{2}+d r^{2}\right), \quad e^{\Phi}=t^{\alpha} ; \quad \sum_{i} p_{i}=1, \quad \sum_{i} p_{i}^{2}=1-\frac{1}{2} \alpha^{2} .
$$


The Kasner exponents $p_{i}$ are constrained as above. We thus see that for constant (undeformed) scalar $\Phi$ with $\alpha=0$, the Kasner space is necessarily anisotropic: the $p_{i}$ cannot all be equal. In this case, the gauge theory lives on a time-dependent space but the gauge coupling is not time-dependent. An isotropic AdS-Kasner singularity

$$
d s^{2}=\frac{R_{\mathrm{AdS}}^{2}}{r^{2}}\left(-d t^{2}+t^{2 / 3} d x_{i}^{2}+d r^{2}\right), \quad e^{\Phi}=t^{2 / \sqrt{3}},
$$

has all Kasner exponents equal: this requires a nontrivial time-dependent dilaton source $\Phi$ as well. More general backgrounds can also be found involving AdS-FRW and AdS-BKL spacetimes [26, 27]: these all have spacelike singularities. There are also backgrounds with null singularities: in these cases, the dual appears to be weakly coupled gauge theory [25], modulo some caveats (section 5). Similar backgrounds arise for $A d S_{4} \times X^{7}$ deformations although the details differ slightly: e.g. the isotropic $A d S_{4}$-Kasner background has $p_{i}=\frac{1}{2}$ and $\frac{\alpha^{2}}{2}=\frac{1}{2}$ giving

$$
d s^{2}=\frac{R_{\mathrm{AdS}}^{2}}{r^{2}}\left(-d t^{2}+t d x_{i}^{2}+d r^{2}\right), \quad e^{\Phi}=t .
$$

These also contain a Big-Crunch singularity as for the $A d S_{5}$ case, but with different exponents controlling the approach to the singularity (and a nontrivial $\Phi$ necessarily). In this case the scalar $\Phi$ arises from the 4-form flux after compactification on some 7-manifold $X^{7}$ : see [60] for more details on the 11-dim supergravity constructions of these backgrounds.

The above backgrounds are solutions to Einstein-dilaton gravity with negative cosmological constant $\Lambda<0$ : these arise as consistent truncations of IIB string theory or M-theory on $A d S \times S$ spaces, the flux giving rise to the effective cosmological constant.

We will refer to the scalar $\Phi$ as the "string dilaton" here (although it is only in the $A d S_{5} / C F T_{4}$ case that $\Phi$ controls the string coupling). The word dilaton will refer to the scalar that plays the role of the 2-dim dilaton, arising from Kaluza-Klein compactification from higher dimensions (this controls the transverse area of the higher dimensional space).

\section{Redux to 2d dilaton gravity: Big-Crunch attractors}

In what follows, we would like to explore Kaluza-Klein reductions of these models of BigBang/Crunch singularities down to two bulk dimensions. See e.g. [61-64] for a review of 2-dim theories obtained from dimensional reduction (and [65]-[73] for some previous work on $2 \mathrm{~d}$ cosmologies in part motivated by the CGHS model of 2-dim black holes [74]). 2-dim dilaton gravity has been revisited in [75] in the context of $A d S_{2}$ holography. We are considering higher dimensional theories of Einstein gravity with a scalar $\Phi$ and a potential $V$, the action being of the form

$$
S=\frac{1}{16 \pi G_{D}} \int d^{D} x \sqrt{-g^{(D)}}\left(\mathcal{R}-\frac{1}{2}(\partial \Phi)^{2}-V\right) .
$$

We allow the potential $V$ to also contain metric data, i.e. it is a function $V(g, \Phi)$. These sorts of theories (which we will expand on later) were considered in $[76,77]$ whose conventions we are following. 
Let us take the reduction ansatz on some compact space $M^{D-2}$ to be

$$
d s^{2} \equiv g_{\mu \nu}^{(2)} d x^{\mu} d x^{\nu}+X^{\frac{4}{D-2}} d \sigma_{D-2}^{2}, \quad D=d_{i}+2
$$

It is convenient to define $d_{i}=D-2$ as the "transverse" part of the bulk spacetime, apart from the 2-dimensional piece (which will contain time $t$ ): in theories with holographic duals, $d_{i}$ will become the boundary spatial dimension. With this form of the 2-dim dilaton $X$, the 2-dim dilaton gravity action is of the form $\int\left(X^{2} \mathcal{R}^{(2)}+\ldots\right)$ : also the dilaton $X^{2}$ is essentially the transverse area $g_{i i}^{D-2}=X^{2}$. This ansatz for $M^{D-2}$ being a torus $T^{D-2}$ implies translation/rotational invariance in the spatial directions in the higher dimensional background (as is the case for various relativistic backgrounds such as AdS or conformally AdS, or nonrelativistic ones such as hyperscaling violating Lifshitz).

In detail, the reduction ansatz (3.2) gives

$$
S=\frac{1}{16 \pi G_{2}} \int d^{2} x \sqrt{-g^{(2)}}\left(X^{2} \mathcal{R}^{(2)}+\frac{D-3}{D-2} \frac{\left(\partial X^{2}\right)^{2}}{X^{2}}-\frac{1}{2} X^{2}(\partial \Phi)^{2}-V X^{2}\right) .
$$

A total derivative term that arises in this reduction cancels with a corresponding term from reduction of the Gibbons Hawking boundary term. In [76, 77], as in [75], an additional Weyl transformation was performed to absorb the dilaton kinetic term in the 2-dim Ricci scalar: note that a Weyl transform acts as

$$
\sqrt{-g^{(2)}}\left[\Psi^{2} \mathcal{R}^{(2)}+\lambda(\partial \Psi)^{2}\right] \quad \stackrel{g_{a b}^{(2)}=\Psi^{-\alpha / 2} g_{a b}}{\longrightarrow} \quad \sqrt{-g}\left[\Psi^{2} \mathcal{R}+(\lambda-\alpha)(\partial \Psi)^{2}\right]
$$

Thus defining

$$
g_{\mu \nu}=X^{\frac{2(D-3)}{D-2}} g_{\mu \nu}^{(2)}
$$

removes the kinetic term for the dilaton $X$ in (3.3) above. The Weyl transformation factors cancel in the derivative terms $(\partial \Phi)^{2}=g^{\mu \nu} \partial_{\mu} \Phi \partial_{\nu} \Phi$ with $\sqrt{-g}$ and the factor in $V$ gives $X^{-2 \frac{D-3}{D-2}+2}$. The resulting action is in agreement with e.g. [63, 64, 78] on the s-wave reduction of Einstein gravity (dropping a term from the sphere curvature) and others.

Thus finally we obtain the action

$$
S=\frac{1}{16 \pi G_{2}} \int d^{2} x \sqrt{-g}\left(X^{2} \mathcal{R}-\frac{1}{2} X^{2}(\partial \Phi)^{2}-U(X, \Phi)\right)
$$

with $U=V X^{2 /(D-2)}$ for the reduction of (3.1) above.

More generally, $U(X, \Phi)$ is some general dilaton potential coupling the dilaton $X$ to the scalar $\Phi$. The equations of motion from this general action are

$$
\begin{aligned}
g_{\mu \nu} \nabla^{2} X^{2}-\nabla_{\mu} \nabla_{\nu} X^{2}+\frac{g_{\mu \nu}}{2}\left(\frac{X^{2}}{2}(\partial \Phi)^{2}+U\right)-\frac{X^{2}}{2} \partial_{\mu} \Phi \partial_{\nu} \Phi & =0, \\
\mathcal{R}-\frac{1}{2}(\partial \Phi)^{2}-\frac{\partial U}{\partial\left(X^{2}\right)} & =0, \\
\frac{1}{\sqrt{-g}} \partial_{\mu}\left(\sqrt{-g} X^{2} \partial^{\mu} \Phi\right)-\frac{\partial U}{\partial \Phi} & =0,
\end{aligned}
$$


Without loss of generality, we can employ conformal gauge and take the 2-dim metric as

$$
d s^{2}=g_{\mu \nu} d x^{\mu} d x^{\nu}=e^{f(t, r)}\left(-d t^{2}+d r^{2}\right) .
$$

Then $\Gamma_{t t}^{t}=\Gamma_{r r}^{t}=\Gamma_{t r}^{r}=\frac{1}{2} \dot{f}, \quad \Gamma_{t r}^{t}=\Gamma_{r r}^{r}=\Gamma_{t t}^{r}=\frac{1}{2} f^{\prime}$ and $\mathcal{R}=e^{-f(t, r)}\left(\ddot{f}-f^{\prime \prime}\right)$.

Combining the various components of the Einstein equations and simplifying gives

$$
\begin{aligned}
(t r) & \partial_{t} \partial_{r} X^{2}-\frac{1}{2} f^{\prime} \partial_{t} X^{2}-\frac{1}{2} \dot{f} \partial_{r} X^{2}+\frac{X^{2}}{2} \dot{\Phi} \Phi^{\prime} & =0, \\
(r r+t t) & -\partial_{t}^{2} X^{2}-\partial_{r}^{2} X^{2}+\dot{f} \partial_{t} X^{2}+f^{\prime} \partial_{r} X^{2}-\frac{X^{2}}{2}(\dot{\Phi})^{2}-\frac{X^{2}}{2}\left(\Phi^{\prime}\right)^{2} & =0 \\
(r r-t t) & -\partial_{t}^{2} X^{2}+\partial_{r}^{2} X^{2}+e^{f} U & =0 \\
(X) & \left(\ddot{f}-f^{\prime \prime}\right)-\frac{1}{2}\left(-(\dot{\Phi})^{2}+\left(\Phi^{\prime}\right)^{2}\right)-e^{f} \frac{\partial U}{\partial\left(X^{2}\right)} & =0 \\
(\Phi) & -\partial_{t}\left(X^{2} \partial_{t} \Phi\right)+\partial_{r}\left(X^{2} \partial_{r} \Phi\right)-e^{f} \frac{\partial U}{\partial \Phi} & =0
\end{aligned}
$$

Here we have combined the $t t$ - and $r r$-components of the Einstein equations in (3.7): this form turns out to be instructive and useful in what follows.

We expect that in the asymptotic regions, the backgrounds become "nearly" static: the fields are slowly varying. So turning the time derivatives off in these regions gives

$$
\begin{aligned}
-\partial_{r}^{2} X^{2}+f^{\prime} \partial_{r} X^{2}-\frac{X^{2}}{2}\left(\Phi^{\prime}\right)^{2} & =0, & \partial_{r}^{2} X^{2}+e^{f} U & =0, \\
-f^{\prime \prime}-\frac{1}{2}\left(\Phi^{\prime}\right)^{2}-e^{f} \frac{\partial U}{\partial\left(X^{2}\right)} & =0, & \partial_{r}\left(X^{2} \partial_{r} \Phi\right)-e^{f} \frac{\partial U}{\partial \Phi} & =0 .
\end{aligned}
$$

These equations define the asymptotic regions describing the background: the $r$-behaviour is subject to these.

Now let us assume some Big-Crunch singularity arises: near such a singularity, there is rapid time variation, approaching a divergence. Thus taking the time derivative terms to be dominant (dropping all the other terms) gives the near singularity behaviour described by

$$
-\partial_{t}^{2} X^{2}+\dot{f} \partial_{t} X^{2}-\frac{X^{2}}{2}(\dot{\Phi})^{2} \sim 0, \quad-\partial_{t}^{2} X^{2} \sim 0, \quad \ddot{f}+\frac{1}{2}(\dot{\Phi})^{2} \sim 0, \quad-\partial_{t}\left(X^{2} \partial_{t} \Phi\right) \sim 0 .
$$

Interestingly, this appears "universal": the dilaton potential $U$ governing the asymptotic behaviour of the background has disappeared. Combining these equations gives

$$
-\partial_{t}^{2} X^{2} \sim 0, \quad \partial_{t}\left(X^{2} \partial_{t} f\right) \sim 0, \quad \ddot{f}+\frac{1}{2}(\dot{\Phi})^{2} \sim 0, \quad \partial_{t}\left(X^{2} \partial_{t} \Phi\right) \sim 0 .
$$

Taking $X^{2} \sim t^{k}$ gives $k(k-1)=0 . \quad(k=0$, consistent with $a=0, \alpha=0$, is timeindependent) Solving these shows that the cosmological singularity is governed by a "universal" subsector:

$$
X^{2} \sim t, \quad e^{f} \sim t^{a}, \quad e^{\Phi} \sim t^{\alpha} ; \quad a=\frac{\alpha^{2}}{2} .
$$

These can be thought of as a Big-Bang $(t>0)$ or -Crunch $(t<0)$ : we will often find it convenient to refer to a Crunch, but will continue to use $t$, not bothering about its sign. 
This universal subsector is like a cosmological attractor: the behaviour near the singularity, when it exists, has this universal scaling behaviour. The question of its existence can only be answered by a detailed analysis of the above equations in full. This full analysis will determine the precise values of the exponents $a, \alpha$, and in particular the tr-equation which couples the $t$ - and $r$-sectors will answer the question of existence. We will carry out this detailed analysis later (section 4 ).

Flat space Kasner cosmological singularities. The universal subsector (3.13) above is in fact the reduction to 2-dimensions of the well-known Kasner cosmological singularities restricted to the "mostly" isotropic subcase, as we will see now. These arise in Einstein gravity coupled to a scalar $\Phi$, with action (3.1) and no potential $V=0$. Reduction gives the 2-dim action (3.6) with $U=0$. The Kasner exponents $p_{i}$ are related to $a, \alpha$ above.

The "mostly isotropic" Kasner cosmology in $D$-dimensions sourced by a scalar $\Phi$ is

$$
\begin{aligned}
d s_{D}^{2} & =-d t^{2}+t^{2 p_{1}} d x_{1}^{2}+t^{2 p_{2}} \sum_{i=2}^{D-1} d x_{i}^{2}, \quad e^{\Phi}=t^{\alpha}, \\
p_{1}+(D-2) p_{2} & =1, \\
p_{1}^{2}+(D-2) p_{2}^{2} & =1-\frac{1}{2} \alpha^{2} .
\end{aligned}
$$

This is a 1-parameter family of Big-Crunch singularities, parametrized by the scalar exponent $\alpha$. When $\alpha=0$, these have $p_{1}=-\frac{1}{3}, p_{2}=\frac{2}{3}$ when $D=4$ : this is the behaviour near the spacelike curvature singularity in the interior of the Schwarzschild black hole in 4-dim. See [51] for a recent discussion.

Upon reduction on $T^{D-2} \equiv\left\{x_{2}, \ldots, x_{D-1}\right\}$, this gives, using (3.2), (3.5),

$$
X^{\frac{4}{D-2}}=t^{2 p_{2}}, \quad d s^{2}=X^{\frac{2(D-3)}{D-2}}\left(-d t^{2}+t^{2 p_{1}} d x_{1}^{2}\right)=t^{2 p_{1}+(D-3) p_{2}}\left(-d t^{2} / t^{2 p_{1}}+d x_{1}^{2}\right) .
$$

Redefining gives

$$
T=t^{1-p_{1}}: \quad X^{2}=T, \quad d s^{2}=T^{\left((D-3) p_{2}+2 p_{1}\right) /\left(1-p_{i}\right)}\left(-d T^{2}+d x_{1}^{2}\right), \quad e^{\Phi}=T^{\alpha /\left(1-p_{1}\right)} .
$$

It can then be checked using (3.14) that these exponents satisfy (3.13). The parametrization (3.13) of (3.14) is just more convenient for this mostly isotropic subclass of singularities it describes.

These backgrounds do not have any spatial dependence: so the full equations (3.9) in fact reduce to (3.11), the asymptotic region equations (3.10) being trivial.

AdS-Kasner singularities and redux. The higher dimensional AdS theories we have been discussing are deformations of $A d S \times S$. The bulk theories are solutions to Einstein gravity with the string dilaton $\Phi$, with action

$$
\begin{aligned}
& S=\frac{1}{16 \pi G_{D}} \int d^{D} x \sqrt{-g^{(D)}}\left(\mathcal{R}-2 \Lambda-\frac{1}{2}(\partial \Phi)^{2}\right) \\
& \longrightarrow \frac{1}{16 \pi G_{2}} \int d^{2} x \sqrt{-g}\left(X^{2} \mathcal{R}-2 \Lambda X^{2 /(D-2)}-\frac{1}{2} X^{2}(\partial \Phi)^{2}\right)
\end{aligned}
$$


using the reduction (3.2), (3.5). Setting the AdS scale in section 2 to unity $R_{\text {AdS }}=1$ makes $\Lambda$ also dimensionless: so this $A d S_{D}$ redux gives the 2-dim action (3.6) with

$$
U=2 \Lambda X^{2 / d_{i}}, \quad \Lambda=-\frac{1}{2} d_{i}\left(d_{i}+1\right), \quad D=d_{i}+2,
$$

Here $d_{i}$ is the spatial dimension of the boundary theory, and we have

$$
\begin{aligned}
d s^{2} & =\frac{1}{r^{2}}\left(-d t^{2}+d r^{2}\right)+\frac{t^{2 p}}{r^{2}} \sum_{i} d x_{i}^{2}, \quad e^{\Phi}=t^{\alpha}, \\
p & =\frac{1}{d_{i}}, \quad \alpha=\sqrt{2\left(1-d_{i} p^{2}\right)}=\sqrt{\frac{2\left(d_{i}-1\right)}{d_{i}} .}
\end{aligned}
$$

The exponent $p$ is related to the dimension for these isotropic AdS-Kasner backgrounds using (2.1). This gives

$$
d s_{A d S_{d_{i}+2}}^{2}=\frac{1}{r^{2}}\left(-d t^{2}+d r^{2}\right)+\frac{t^{2 / d_{i}}}{r^{2}} d x_{i}^{2},
$$

so using (3.2), (3.5), gives the 2-dim background,

$$
X^{2} \sim \frac{t}{r^{d_{i}}}, \quad d s^{2}=X^{\frac{2\left(d_{i}-1\right)}{d_{i}}} d s_{2}^{2}=\frac{t^{\left(d_{i}-1\right) / d_{i}}}{r^{d_{i}+1}}\left(-d t^{2}+d r^{2}\right), \quad e^{\Phi}=t^{\sqrt{2\left(d_{i}-1\right) / d_{i}}} .
$$

We see that the behaviour near the singularity $t \sim 0$ is precisely as in (3.13).

The precise value of the exponents $a, \alpha$ is fixed from the asymptotics, using (3.10): for instance, for the time-dependence to cancel in $\partial_{r}^{2} X^{2}+e^{f} U=0$, we require

$$
t^{k} \sim t^{a} t^{k / d_{i}} \Rightarrow a+\frac{k}{d_{i}}=k \Rightarrow a=\frac{d_{i}-1}{d_{i}},
$$

using $k=1$. We will recover this and other solutions from a detailed analysis in what follows.

\section{Solving the 2-dim theory for Big-Crunches}

We would now like to carry out a detailed analysis of backgrounds with cosmological, BigCrunch type, singularities, for theories of the form (3.6): we will discuss various kinds of potentials $U$ that arise from familiar theories under KK-reduction.

Towards this, we take the following scaling-type ansatz

$$
X^{2}=t^{k} r^{m}, \quad e^{f}=t^{a} r^{b}, \quad e^{\Phi}=t^{\alpha} r^{\beta} .
$$

In doing this, we have set relevant lengthscales to unity. For instance, we have set $R_{\mathrm{AdS}}=1$, recasting (2.1) as (3.19): then the metric is dimensionless. It is straightforward to reinstate the lengthscales when necessary.

For time-independent cases, this is in accord with the kinds of power law behaviour in known backgrounds such as AdS or conformally AdS, and also consistent with the asymptotic (time-independent) form of the equations (3.10). For time-dependent situations, our 
intuition is that the power law $t$-behaviour implies a Crunch (or Bang) at some time $t=0$ : so in sense, the ansatz (4.1) is a "near singularity" ansatz describing the behaviour near the cosmological singularity, consistent with the universal near singularity form (3.11), (3.13) of the equations of motion (3.7), (3.9). Juxtaposing the time part is consistent with the apparent separability of the equations into time and space parts, and we will in fact find that this is consistent. However this does not of course include the possibility of more exotic time-dependent backgrounds which do not admit any factorization.

The scaling ansatz (4.1) gives

$$
\dot{f}=\frac{a}{t}, \quad f^{\prime}=\frac{b}{r} \quad \text { and } \quad \dot{\Phi}=\frac{\alpha}{t}, \quad \Phi^{\prime}=\frac{\beta}{r} .
$$

Using this and putting the scaling ansatze (4.1) back in the differential equations (3.9) and requiring that nontrivial solutions exist for all $t, r$, gives a set of algebraic equations for the various exponents. Solving for the exponents then determines the full solution. In a sense, this is akin to the Frobenius series solutions for differential equations, with the additional assumption that it is adequate to focus on the leading monomials in a series e.g. $\sum_{i} t^{m_{i}} r^{n_{i}}$. This is consistent for both the asymptotic region (large $r$ ) and the near singularity region $(t \rightarrow 0)$ where appropriate leading terms can be taken to be good approximations.

In practice, for time-dependent backgrounds, these equations can be solved by first taking the $t$ - and $r$-sectors as decoupled: this yields solutions for the $t$ - and $r$-exponents, which can then be stuck into the tr-equation which is the only equation coupling the $t$ and $r$-sectors. We will see this explicitly below.

\subsection{AdS and conformally AdS backgrounds}

Consider the class of models given by the higher dimensional action

$$
\begin{aligned}
& S=\int d^{d_{i}+2} x \sqrt{-g}\left(\mathcal{R}-\frac{1}{2}(\partial \Phi)^{2}-2 \Lambda e^{\gamma \Phi}\right), \quad \Lambda=-\frac{1}{2}\left(d_{i}+1-\theta\right)\left(d_{i}-\theta\right), \\
& \gamma=\frac{-2 \theta}{\sqrt{2 d_{i}\left(d_{i}-\theta\right)(-\theta)}}, \quad d_{i}=p=D-2, \quad \theta=p-\frac{9-p}{5-p}<0 .
\end{aligned}
$$

For $\theta=0$ these describe conformal branes: $\gamma=0$ and the potential in $\Phi$ is simply the negative cosmological constant term arising from the flux integrated over the transverse sphere. The parameter $\theta$ is called the "hyperscaling violation" exponent: these theories are a subclass of more general hyperscaling violating Lifshitz theories $[79,80]$ which we will discuss later. For nonzero $\theta$, these arise from nonconformal $D p$-branes [81] after dimensional reduction over the transverse sphere $S^{8-p}$ [82]. The known time-independent backgrounds are $(p+2)$-dimensional spaces of the form

$$
d s^{2}=r^{2 \theta / d_{i}}\left(\frac{-d t^{2}+d x_{i}^{2}+d r^{2}}{r^{2}}\right), \quad e^{\Phi}=r^{\sqrt{2\left(d_{i}-\theta\right)\left(-\theta / d_{i}\right)}} .
$$

For $\theta=0$ these give $A d S_{p+2}$ with $\Phi=$ const. Nonzero $\theta$ gives an extra conformal factor and a nontrivial string dilaton $\Phi$ which is required to source such backgrounds: it is also related to the RG running of the dual field theory. Besides $R_{p}$ akin to $R_{\text {AdS }}$ earlier, there 
is a new length scale here $r_{h v}$ which appears in all $\theta$-dependent terms: e.g. the conformal factor is $\left(\frac{r}{r_{h v}}\right)^{2 \theta / d_{i}}$, and likewise for $e^{\Phi}$. It is straightforward to reinstate $R_{p}$ and $r_{h v}$.

The action above is of the form (3.1): under reduction with (3.2), (3.5), we obtain a 2-dimensional dilaton-gravity-matter theory of the form (3.6). The dilaton potential here is

$$
U(X, \Phi)=-c_{1} X^{\frac{2}{d_{i}}} e^{\gamma \Phi}, \quad c_{1}=-2 \Lambda=\left(d_{i}+1-\theta\right)\left(d_{i}-\theta\right) .
$$

For $\gamma=0$, we see that this becomes (3.18) as expected. We have

$$
\frac{\partial U}{\partial\left(X^{2}\right)}=-\frac{c_{1}}{d_{i}}\left(X^{2}\right)^{\frac{1}{d_{i}}-1} e^{\gamma \Phi}, \quad \frac{\partial U}{\partial \Phi}=-\gamma c_{1} X^{\frac{2}{d_{i}}} e^{\gamma \Phi} .
$$

Now using the ansatz (4.1) in (3.9) gives the algebraic equations:

$$
\begin{array}{rr}
(t r) & {\left[m k-\frac{b k}{2}-\frac{a m}{2}+\frac{\alpha \beta}{2}\right] t^{k-1} r^{m-1}=0,} \\
(r r+t t) & {\left[-k(k-1)+a k-\frac{\alpha^{2}}{2}\right] t^{k-2} r^{m}+\left(-m(m-1)+b m-\frac{\beta^{2}}{2}\right) r^{m-2} t^{k}=0,} \\
(r r-t t) & {[-k(k-1)] \frac{r^{2}}{t^{2}}+m(m-1)-c_{1} t^{a+k\left(1 / d_{i}-1\right)+\gamma \alpha} r^{b+m\left(1 / d_{i}-1\right)+\gamma \beta+2}=0,} \\
(X) & -\frac{r^{2}}{t^{2}}\left[a-\frac{\alpha^{2}}{2}\right]+b-\frac{\beta^{2}}{2}+\frac{c_{1}}{d_{i}} t^{a+k\left(1 / d_{i}-1\right)+\gamma \alpha} r^{b+m\left(1 / d_{i}-1\right)+\gamma \beta+2}=0, \\
(\Phi) & {[-\alpha(k-1)] \frac{r^{2}}{t^{2}}+\beta(m-1)+\gamma c_{1} t^{a+k\left(1 / d_{i}-1\right)+\gamma \alpha} r^{b+m\left(1 / d_{i}-1\right)+\gamma \beta+2}=0 .}
\end{array}
$$

In the above equations, we have demarcated the time-dependent terms in square brackets for convenience. Then for time-independent solutions, all the square bracket terms above must vanish: in particular the (tr)-equation is automatically satisfied while the other equations give nontrivial constraints on the $r$-behaviour.

As stated earlier, for time-dependent backgrounds, we first take the $t$ - and $r$-sectors as decoupled, thereby obtaining solutions for the $t$ - and $r$-exponents. These can then be stuck into the tr-equation which is the only equation coupling the $t$ - and $r$-sectors.

Thus, from the $r$ - and $t$-equations above, requiring that the various $t^{A} r^{B}$ terms either cancel in groups or vanish (for non-matching exponents), we find the following nontrivial family of solutions, characterized by relations between the exponents $(k, a, \alpha)$ and $(m, b, \beta)$ and the parameters $d_{i}, \theta, c_{1}$ of the theory:

$$
\begin{array}{rlrl}
(r) \quad b=\frac{\beta^{2}}{2}-\frac{m(m-1)}{d_{i}} ; & b m & =\frac{\beta^{2}}{2}+m(m-1) ; & b+\frac{m\left(1-d_{i}\right)}{d_{i}}+\gamma \beta=-2 ; \\
(\beta+\gamma m)(m-1)=0 ; & m(m-1) & =c_{1} ; \\
(t) \quad & a & =\frac{\alpha^{2}}{2} ; \alpha(k-1)=0 ; & a+\frac{k\left(1-d_{i}\right)}{d_{i}}+\gamma \alpha=0 .
\end{array}
$$

The $(t)$-equations arise from the square bracket terms in (4.7). The last equation here arises from noting that the exponent of $t$ in the terms containing $c_{1}$ must independently vanish for a nontrivial solution to exist for all $t, r$ (thus matching the vanishing $t$-exponent in the other terms), as stated above. 
Likewise the $(r)$-equations arise from noting that the terms outside the square brackets must vanish for generic $t^{A} r^{B}$ terms: in particular this also requires that the exponent of $r$ in the terms containing $c_{1}$ must vanish (as for $t$ above), leading to the first two $(r)$-equations. Finally we have used the equation $m(m-1)=c_{1}$ from the $(r r-t t)$-equation in (4.7) to recast the above equations (4.8), (4.9), in a suggestive and useful manner.

Time-independent backgrounds. We will first discuss time-independent solutions, recovering the familiar AdS and nonconformal $D p$-brane backgrounds. This also serves as a check of these equations and our scheme of analysing them. In this case, we have $k=0, a=0, \alpha=0$ : the last equation in (4.9) is in fact not applicable since there is no $t$-exponent in the absence of time-dependence. These imply that the $t r$-equation is automatically satisfied.

Massaging the set of relations between $b, \beta, \gamma$ in (4.8) having eliminated $c_{1}$ as stated above shows that the relations are not all independent, allowing for consistent nontrivial solutions. The first two equations in (4.8) parametrize $b, \beta$ in terms of $m$ : then using $\gamma$ from (4.3) gives

$$
b=\frac{m\left(1+d_{i}\right)}{d_{i}}, \quad \beta=-m \gamma=\sqrt{\frac{2 m\left(m+d_{i}\right)}{d_{i}}}, \quad m=-d_{i}+\theta .
$$

Note $m<0$, since $\theta \leq d_{i}$ from the entropy-temperature relation $S \sim V_{d_{i}} T^{d_{i}-\theta}$ and specific heat positivity [82]. Note also that $m$ obtained from $m(m-1)=c_{1}$ (using (4.5)) gives

$$
m^{2}-m-\left(d_{i}+1-\theta\right)\left(d_{i}-\theta\right)=0 \quad \Rightarrow \quad m=-\left(d_{i}-\theta\right) \text { or }\left(d_{i}-\theta+1\right) .
$$

The first $m$ value corresponds to non-normalizable modes in the higher dimensional theory while $m=d_{i}-\theta+1$ are normalizable modes. Of these, we see that only the non-normalizable $m$ value satisfies all the equations in (4.8), (4.9). Note also that the possible value $m=1$ satisfies all equations except for $c_{1}=m(m-1)$ with nonzero $c_{1}$.

These in fact recover the reduction of the backgrounds in (4.4), using (3.2), (3.5) and (4.1). For instance, $X^{4 / d_{i}}=r^{2 \theta / d_{i}-2}$ gives $m=-d_{i}+\theta$, while $\beta=\sqrt{2\left(d_{i}-\theta\right)\left(-\theta / d_{i}\right)}$ matches with the $e^{\Phi}$ exponent. Likewise $b$ matches with the $e^{f}$ exponent after noting that the piece $\frac{m\left(1-d_{i}\right)}{d_{i}}$ in (4.8) arises from the Weyl transformation (3.5) (likewise for the $\frac{k\left(1-d_{i}\right)}{d_{i}}$ in $(4.9))$.

Now coming to cosmological solutions with time-dependence, we see the universal sector $k=1, a=\frac{\alpha^{2}}{2}$ from the first two $(t)$-equations, while the last equation fixes the precise value of $a, \alpha$. These show a Big-Crunch type cosmological singularity at $t=0$ with $X^{2} \sim t$ always. We will now discuss various subcases here.

$\boldsymbol{A d} \boldsymbol{S}_{\boldsymbol{D}}$ Big-Crunch redux. Here $\theta=0$ and so $\gamma=0=\beta$. Then (4.10) and the time-dependent subsector (4.9) can be seen to give the 2-dim background (4.1) with the exponents

$$
\begin{array}{ll}
\theta=0=\gamma, & \beta=0, \quad m=-d_{i}, \quad b=-\left(d_{i}+1\right), \\
k=1, & a=\frac{\alpha^{2}}{2}=\frac{d_{i}-1}{d_{i}} .
\end{array}
$$


The $t r$-equation $m-\frac{b}{2}-\frac{a m}{2}-\frac{\alpha \beta}{2}=0$ is explicitly seen to be satisfied. Thus we recover the AdS Kasner cosmological singularities (3.19) in the form (3.21) after reduction to 2dimensions, noting $D=d_{i}+2$. We have chosen $\alpha>0$ so $e^{\Phi} \rightarrow 0$ near the singularity.

Nonconformal $\boldsymbol{D} \boldsymbol{p}$-brane Big-Crunch redux. Now we have $\theta \neq 0$, the higher dimensional theories being (4.3). The time-sector (4.9) now contains $\gamma$ as well, and can be solved for $a, \alpha$. The $r$-sector, decoupled from the $t$-sector, can be solved as for the timeindependent case in (4.10). Putting these together gives the 2-dim background (4.1) with exponents

$$
\begin{array}{llll}
m=-\left(d_{i}-\theta\right), & b=-\frac{\left(d_{i}-\theta\right)\left(1+d_{i}\right)}{d_{i}}, & \beta & =-m \gamma=\sqrt{\frac{2\left(d_{i}-\theta\right)(-\theta)}{d_{i}}}, \\
k=1, & a=\frac{\alpha^{2}}{2}, & \alpha=-\gamma \pm \sqrt{\gamma^{2}+\frac{2\left(d_{i}-1\right)}{d_{i}}}
\end{array}
$$

(recall $\theta<0, \gamma>0$, from (4.3)) The tr-equation, using (4.8), (4.9), then gives

$$
m-\frac{b}{2}-\frac{a m}{2}+\frac{\alpha \beta}{2}=m+\frac{m}{2}\left(\frac{1-d_{i}}{d_{i}}+\gamma \alpha\right)-\frac{1}{2}\left(-2-m \frac{1-d_{i}}{d_{i}}+m \gamma^{2}\right)-\frac{m \gamma \alpha}{2}
$$

We see that $\alpha$ drops out of this entirely: the remaining terms can be seen to vanish, using the exponents (4.14) above: thus the tr-equation is satisfied here as well.

Now using (3.2) and undoing the Weyl transformation (3.5) gives the higher dimensional theory corresponding to the 2-dim Big-Crunch (4.1) with exponents above: we obtain

$$
d s^{2}=\frac{e^{f}}{X^{\frac{2\left(d_{i}-1\right)}{d_{i}}}}\left(-d t^{2}+d r^{2}\right)+X^{\frac{4}{d_{i}}} d x_{i}^{2}=\frac{r^{\frac{2 \theta}{d_{i}}}}{r^{2}}\left(\frac{-d t^{2}+d r^{2}}{t^{\gamma \alpha}}+t^{\frac{2}{d_{i}}} d x_{i}^{2}\right), e^{\Phi}=t^{\alpha} r^{\sqrt{2\left(d_{i}-\theta\right)\left(\frac{-\theta}{d_{i}}\right)}},
$$

noting that the $r$-exponents are the same as in the time-independent backgrounds stated earlier. Note that the $t r$-part of the spacetime has $t$-behaviour opposite to that of $e^{\Phi}$ since $\gamma>0$ : thus taking the positive sign in $\alpha$ above so that $\alpha>0$ leads to $e^{\Phi} \rightarrow 0$ as $t \rightarrow 0$ but the $t r$-part expands (although the $x_{i}$-part crunches). Conventionally this is what would be called a Big-Crunch (as in the AdS case earlier).

\subsection{Hyperscaling violating Lifshitz asymptotics}

We now consider another class of nonrelativistic theories, hyperscaling violating Lifshitz (hvLif) ones. Consider the action

$$
\begin{array}{rlrl}
S & =\int d^{d_{i}+2} x \sqrt{-g}\left(\mathcal{R}-\frac{1}{2}(\partial \Phi)^{2}-\frac{1}{4} Z(\Phi) F^{2}-2 \Lambda e^{\gamma \Phi}\right) \\
Z(\Phi) & =e^{-\lambda \Phi}, & \Lambda & =-\frac{1}{2}\left(d_{i}+z-\theta\right)\left(d_{i}+z-\theta-1\right) \\
\gamma & =\frac{-2 \theta}{\sqrt{2 d_{i}\left(d_{i}-\theta\right)\left(d_{i} z-d_{i}-\theta\right)}}, & \lambda & =\frac{-2\left(\theta+d_{i}\left(d_{i}-\theta\right)\right)}{\sqrt{2 d_{i}\left(d_{i}-\theta\right)\left(d_{i} z-d_{i}-\theta\right)}} .
\end{array}
$$


These are Einstein-Maxwell-Dilaton theories, containing gravity (and $\Lambda<0$ ) sourced by a gauge field $F$ and a scalar $\Phi$. Such theories have been actively studied in the last several years in the context of "AdS/CMT" towards understanding nonrelativistic generalizations of holography and condensed-matter-type applications. See e.g. [79, 80] for a review of some of these developments (our conventions for the higher dimensional theories in this section are as in [83]). These admit hvLif spacetimes,

$$
\begin{aligned}
d s^{2} & =\rho^{2 \theta / d_{i}}\left(-\frac{d t^{2}}{\rho^{2 z}}+\frac{d x_{i}^{2}+d \rho^{2}}{\rho^{2}}\right), \quad A_{1, t}=-\sqrt{\frac{2(z-1)}{d_{i}+z-\theta}} \frac{1}{\rho^{d_{i}+z-\theta}}, \\
e^{\Phi} & =\rho^{\sqrt{2\left(d_{i}-\theta\right)\left(z-\theta / d_{i}-1\right)}}
\end{aligned}
$$

where $z$ is the Lifshitz exponent and $\theta$ the hyperscaling violating one. In the subsector with the gauge field background profiles taken on-shell, these theories give theories with effective action of the form (3.1): this is similar in spirit to the 5 -form field strength giving rise to an effective cosmological constant in 5-dimensions for a D3-brane stack. This is described in greater detail in [76, 77]. Dimensional reduction of these effective theories using (3.2), (3.5), leads to 2-dimensional dilaton-gravity-matter theories of the form (3.6) with the potential taking the form

$$
U=X^{2 / d_{i}}\left(-c_{1} e^{\gamma \Phi}+\frac{c_{2}}{X^{4}} e^{\lambda \Phi}\right)
$$

For $z=1$, these are conformally AdS spaces of the form (4.4) discussed earlier: in this case, $c_{2}$ vanishes.

With the potential (4.19), we obtain the following equations from (3.9) using the scaling ansatz (4.1):

$$
\begin{array}{r}
(t r) \quad\left[m k-\frac{b k}{2}-\frac{a m}{2}+\frac{\alpha \beta}{2}\right] t^{k-1} r^{m-1}=0, \\
(r r+t t) \quad\left[-k(k-1)+a k-\frac{\alpha^{2}}{2}\right] t^{k-2} r^{m}+\left(-m(m-1)+b m-\frac{\beta^{2}}{2}\right) r^{m-2} t^{k}=0, \\
(r r-t t) \quad[-k(k-1)] \frac{r^{2}}{t^{2}}+m(m-1)-c_{1} t^{a+k\left(1 / d_{i}-1\right)+\gamma \alpha} r^{b+m\left(1 / d_{i}-1\right)+\gamma \beta+2} \\
+c_{2} t^{a+k\left(1 / d_{i}-3\right)+\lambda \alpha} \times r^{b+m\left(1 / d_{i}-3\right)+\lambda \beta+2}=0, \\
(X) \quad-\frac{r^{2}}{t^{2}}\left[a-\frac{\alpha^{2}}{2}\right]+b-\frac{\beta^{2}}{2}+\frac{c_{1}}{d_{i}} t^{a+k\left(1 / d_{i}-1\right)+\gamma \alpha} r^{b+m\left(1 / d_{i}-1\right)+\gamma \beta+2} \\
-c_{2}\left(\frac{1}{d_{i}}-2\right) t^{a+k\left(1 / d_{i}-3\right)+\lambda \alpha} r^{b+m\left(1 / d_{i}-3\right)+\lambda \beta+2}=0, \\
(\Phi) \quad[-\alpha(k-1)] \frac{r^{2}}{t^{2}}+\beta(m-1)+\gamma c_{1} t^{a+k\left(1 / d_{i}-1\right)+\gamma \alpha} r^{b+m\left(1 / d_{i}-1\right)+\gamma \beta+2} \\
-\lambda c_{2} t^{a+k\left(1 / d_{i}-3\right)+\lambda \alpha} r^{b+m\left(1 / d_{i}-3\right)+\lambda \beta+2}=0 .
\end{array}
$$

For $c_{2}=0$, these reduce to (4.7). As in that case, we have demarcated the time-dependent terms in square brackets. These and the other equations give the following algebraic equations between the various exponents $(k, a, \alpha)$ and $(m, b, \beta)$ and the parameters $c_{1}, c_{2}, \gamma, \lambda$ 
of these theories:

$$
m k-\frac{b k}{2}-\frac{a m}{2}+\frac{\alpha \beta}{2}=0
$$

(r) $b m=\frac{\beta^{2}}{2}+m(m-1), \quad b+m\left(\frac{1-d_{i}}{d_{i}}\right)+\gamma \beta+2=0, \quad(\lambda-\gamma) \beta=2 m$,

$$
\begin{gathered}
b-\frac{\beta^{2}}{2}-m(m-1)\left(\frac{1}{d_{i}}-2\right)-2 c_{1}=0, \quad \beta(m-1)+\gamma c_{1}-\lambda c_{2}=0, \\
c_{2}=c_{1}-m(m-1),
\end{gathered}
$$

$(t) \quad-k(k-1)+a k-\frac{\alpha^{2}}{2}=0, \quad k(k-1)=0, \quad a=\frac{\alpha^{2}}{2}, \quad \alpha(k-1)=0$,

$$
a+\left(\frac{1}{d_{i}}-1\right)+\gamma \alpha=0, \quad a+\left(\frac{1}{d_{i}}-3\right)+\lambda \alpha=0 .
$$

These are obtained, as before, by requiring the exponents of various $t^{P} r^{Q}$-terms to be equal for a nontrivial solution valid for all $t, r$, implying that various $t$-exponents independently vanish. Note that, compared with (4.7), (4.8), (4.9), we obtain more constraining equations in this case, stemming from the $c_{2}$ term. In the above, the last equation in (4.22) is obtained by combining the second one there with a similar equation $b+m\left(\frac{1-3 d_{i}}{d_{i}}\right)+\lambda \beta+2=0$ arising from the $r$-exponent in the $c_{2}$-term in the $(r r-t t)-,(X)$ - and $(\Phi)$-equations in (4.20).

As in (4.8), (4.9), we have grouped the $r$ - and $t$-equations separately: the $(t r)$-equation couples them.

Restricting to the time-independent case, we ignore (4.21), (4.25), thereby obtaining the equations (4.22), (4.23), (4.24). The 3 equations in (4.22) can be solved (and simplified in e.g. Mathematica), using the $\gamma, \lambda$-values in (4.17) to give

$$
m=\frac{-d_{i}+\theta}{z}, \quad \beta=\frac{\sqrt{2\left(d_{i}-\theta\right)\left(z-1-\theta / d_{i}\right)}}{z}, \quad b=\frac{\theta+d_{i}\left(1-d_{i}+\theta-2 z\right)}{d_{i} z} .
$$

It can be checked that these also solve the equation obtained by eliminating $c_{2}$ using (4.24) and then $c_{1}$ from the two equations in (4.23) above. Using (4.26), $c_{1}, c_{2}$ are found as

$$
c_{1}=\frac{\left(d_{i}+z-\theta\right)\left(d_{i}+z-\theta-1\right)}{z^{2}}=-\frac{2 \Lambda}{z^{2}}, \quad c_{2}=\frac{(z-1)\left(d_{i}+z-\theta\right)}{z^{2}},
$$

with $\Lambda$ in (4.17). To compare with the reduction (3.2), (3.5), of hvLif backgrounds, write (4.18) as

$$
d s^{2}=\rho^{2 \theta / d_{i}-2 z}\left(-d t^{2}+\rho^{2 z-2} d \rho^{2}\right)+\rho^{2 \theta / d_{i}-2} d x_{i}^{2} \stackrel{r \sim \rho^{z} / z}{\longrightarrow} \quad r^{\#}\left(-d t^{2}+d r^{2}\right)+r^{\#} d x_{i}^{2} .
$$

These can be seen to match.

Cosmological solutions. These are more intricate and constrained, since the $r$ - and $t$-exponents are now apparently coupled by the $(t r)$-equation which is more constraining in this case. From the last equation in (4.22) and (4.25), we obtain

$$
(\lambda-\gamma) \beta=2 m, \quad(\lambda-\gamma) \alpha=2 \quad \Rightarrow \quad \beta=m \alpha .
$$


Then the first equation in (4.22) alongwith $a=\frac{\alpha^{2}}{2}$ from (4.25) gives

$$
b m=m^{2} a+m(m-1) \quad \Rightarrow \quad b=m(a+1)-1,
$$

taking $m \neq 0$. Sticking these in the $(t r)$-equation (4.21) then gives

$$
\begin{aligned}
& m-\frac{m(a+1)-1}{2}-\frac{a m}{2}+m a=0 \quad \Rightarrow \quad m=-1 . \\
& \Rightarrow \quad m=-1, \quad \beta=-\alpha=-\frac{2}{\lambda-\gamma}, \quad b=-a-2 \text {. }
\end{aligned}
$$

Then the second equation in (4.23) alongwith (4.24) gives

$$
c_{1}-c_{2}=2, \quad 2 \alpha+\gamma c_{1}-\lambda c_{2}=0 \quad \Rightarrow \quad c_{1}=\frac{2(\alpha+\lambda)}{\lambda-\gamma}, \quad c_{2}=\frac{2(\alpha+\gamma)}{\lambda-\gamma} .
$$

Using these in the first equation in (4.23) alongwith (4.32), after some simplification, gives

$$
\beta=\lambda\left(\frac{1}{d_{i}}-1\right)-\gamma\left(\frac{1}{d_{i}}-3\right)
$$

Note that consistency of this $\beta$ value with that in (4.32) gives a nontrivial relation between the parameters $\gamma, \lambda$ of these theories: see below. Finally note that using $a=\frac{\alpha^{2}}{2}$ in the two equations in the second line in (4.25) gives two quadratics, with solutions

$$
\alpha=-\gamma-\sqrt{\gamma^{2}+\frac{2\left(d_{i}-1\right)}{d_{i}}}, \quad \text { and } \quad \alpha=-\lambda-\sqrt{\lambda^{2}+\frac{2\left(3 d_{i}-1\right)}{d_{i}}},
$$

which are consistent only if equal: this after some simplification can be shown to give

$$
\lambda\left(\frac{1}{d_{i}}-1\right)-\gamma\left(\frac{1}{d_{i}}-3\right)=\frac{-2}{\lambda-\gamma} .
$$

This is the same nontrivial condition on $\gamma, \lambda$, as the one from $\beta$ stated above. Taking this as a nontrivial quadratic relation in $\lambda$, we obtain

$$
\lambda=\frac{\left(2 d_{i}-1\right) \gamma-\sqrt{d_{i}^{2}\left(2+\gamma^{2}\right)-2 d_{i}}}{d_{i}-1} .
$$

Now finally, we will check consistency of these exponent values with the parametrization of $\gamma, \lambda$ in (4.17). We have $\lambda-\gamma=\frac{-2 d_{i}\left(d_{i}-\theta\right)}{\sqrt{\cdots}}<0$ since $d_{i}-\theta>0$ from the positivity of specific heat for hvLif theories [82]. This requires $\alpha<0$ and $\beta>0$. This is why we have displayed only the negative signs in the $\alpha$ values as consistent with (4.17): this further implies the negative sign in the $\lambda$ solution above. Now

$$
\lambda-\gamma=\frac{d_{i}}{d_{i}-1}\left(\gamma-\sqrt{\gamma^{2}+2-\frac{2}{d_{i}}}\right)<0
$$

which is consistent with the parametrization (4.17). Additionally, $m=-1$ implies $d_{i}-\theta=z$. 
In particular for $\gamma=0$ and $d_{i}=2$ for simplicity, we have the nontrivial Lifshitz-type cosmological solution (4.1) with

$\theta=0 ; \quad \lambda=-2=\frac{-2 d_{i}^{2}}{\sqrt{2 d_{i}^{3}(z-1)}} \Rightarrow z=2 ; \beta=1=-\alpha, b=-\frac{5}{2}, a=\frac{1}{2}, k=1, m=-1$.

Also $c_{1}=3, c_{2}=1, \Lambda=-6$. It can be checked that these are consistent with all the equations above.

In terms of $\rho=(2 r)^{1 / 2}$, we have (using (3.2) and undoing the Weyl transformation (3.5))

$$
\begin{aligned}
X^{2} & =\frac{t}{\rho^{2}}, \quad e^{f}=\frac{\sqrt{t}}{\rho^{5}}, \quad e^{\Phi}=\frac{\rho^{2}}{t} \\
\Rightarrow \quad d s^{2} & =\frac{e^{f}}{X^{2\left(d_{i}-1\right) / d_{i}}}\left(-d t^{2}+d r^{2}\right)+X^{4 / d_{i}} d x_{i}^{2}=-\frac{d t^{2}}{\rho^{4}}+\frac{d \rho^{2}}{\rho^{2}}+\frac{t}{\rho^{2}} d x_{i}^{2} .
\end{aligned}
$$

In this case, since $\gamma=0$, the $t$-factors in $e^{f}$ and $X^{\#}$ cancel (stemming from (4.25)): more generally there will be $t$-dependence in the $t r$-part as well (as in (4.16)). Then the 2-dim cosmological background (4.1) has exponents exhibiting the universal behaviour $k=1, a=$ $\frac{\alpha^{2}}{2}$ alongwith (4.26), (4.32), (4.35) and (4.36) subject to the parametrization (4.17). This gives the higher dimensional cosmological hvLif spacetime

$$
d s^{2}=\frac{r^{2 \theta / d_{i}}}{r^{2}}\left(\frac{1}{t^{\gamma \alpha}}\left(-\frac{d t^{2}}{r^{2 z}}+d r^{2}\right)+t^{2 / d_{i}} d x_{i}^{2}\right), \quad e^{\Phi}=t^{\alpha} r^{-\alpha},
$$

with $\alpha$ determined, and $z, \theta$ constrained as above.

\subsection{JT gravity}

It is instructive to study $A d S_{2}$ boundary conditions and look for cosmological solutions: it is well known that extremal black holes or branes give rise to $A d S_{2}$ in their throat regions. Performing a dimensional reduction on the transverse space allows focussing on these $A d S_{2}$ dilaton-gravity theories in 2-dimensions: this is the Jackiw-Teitelboim gravity theory $[84,85]$ and has been much studied lately in the context of SYK models and nearly $A d S_{2}$ theories [75, 86-88] (see e.g. [89-91] for some reviews).

The JT gravity theory, with dilaton $X^{2}$, admits $A d S_{2}$ boundary conditions: the action is

$$
S=\frac{1}{16 \pi G_{2}} \int d^{2} x \sqrt{-g}\left[X^{2}(\mathcal{R}+2)\right] .
$$

This is essentially the 2-dimensional action (3.6) with dilaton potential $U=-2 X^{2}$ : this is of the form (4.5) with $c_{1}=2, \gamma=0, d_{i}=2$. For simplicity, we are not including the extra scalar $\Phi$ (it turns out that a free 2-dim scalar $\Phi$ without the $X^{2}$ coupling in (3.6) and additional interaction decouples from the dynamics in this scaling analysis). 
The equations of motion are similar to (3.7), (3.9), and give in this case:

$$
\begin{aligned}
(t r) & \partial_{t} \partial_{r} X^{2}-\frac{1}{2} f^{\prime} \partial_{t} X^{2}-\frac{1}{2} \dot{f} \partial_{r} X^{2}=0, \\
(r r+t t) & -\partial_{t}^{2} X^{2}-\partial_{r}^{2} X^{2}+\dot{f} \partial_{t} X^{2}+f^{\prime} \partial_{r} X^{2}=0, \\
(r r-t t) & -\partial_{t}^{2} X^{2}+\partial_{r}^{2} X^{2}+e^{f} U=0 \\
(X) & \left(\ddot{f}-f^{\prime \prime}\right)-e^{f} \frac{\partial U}{\partial\left(X^{2}\right)}=0 .
\end{aligned}
$$

These are the dilaton equation and the Einstein equations, combined. As before, we look for Big-Crunch type behaviour, defined by large time derivatives, approaching a divergence (thereby dropping the other terms). Then, in contrast with (3.11), (3.13), we see the "near singularity" behaviour as

$$
-\partial_{t}^{2} X^{2}+\dot{f} \partial_{t} X^{2} \sim 0, \quad-\partial_{t}^{2} X^{2} \sim 0, \quad \ddot{f} \sim 0 \quad \Rightarrow \quad f=a t, \quad X^{2}=t .
$$

Instead of (4.1), this suggests the scaling ansatz

$$
X^{2}=t^{k} r^{m}, \quad e^{f}=e^{a t} r^{b} .
$$

Analysing as in the previous cases from redux, we stick in this scaling ansatz in the equations (4.43) and obtain the algebraic equations:

$$
\begin{aligned}
m k t^{k-1} r^{m-1}-\frac{b k}{2} t^{k-1} r^{m-1}-\frac{a m}{2} t^{k} r^{m-1} & =0, \\
-k(k-1) t^{k-2} r^{m}-m(m-1) t^{k} r^{m-2}+a k t^{k-1} r^{m}+b m t^{k} r^{m-2} & =0, \\
-k(k-1) t^{k-2} r^{m}+m(m-1) t^{k} r^{m-2}-2 e^{a t} r^{b} t^{k} r^{m} & =0, \\
\frac{b}{r^{2}}+2 e^{a t} r^{b} & =0 .
\end{aligned}
$$

From the last equation, we have $a=0$ for a nontrivial solution (matching the $t$-exponents): so $b=-2$. From the third equation, we take $k=1$ for a time-dependent solution. Thus

$$
(m+1) r^{m-1}=0, \quad(m(m-1)-2) t r^{m-2}=0, \quad(-m(m-1)+b m) t r^{m-2}=0 .
$$

So finally, we obtain

$$
a=0, \quad b=-2, \quad k=1, \quad m=-1 \quad \Rightarrow \quad X^{2}=\frac{t}{r}, \quad e^{f}=\frac{1}{r^{2}} .
$$

The metric is simply $A d S_{2}$ and the dilaton vanishes at $t=0$, exhibiting a Big-Crunch (while also growing towards the $A d S_{2}$ boundary at $r \sim 0$ ). In cases where JT arises from some higher dimensional reduction, the vanishing dilaton at $t=0$ signals a spacelike singularity where the higher dimensional theory has a Crunch.

It is interesting to compare this with the black hole in JT theory: in lightcone coordinates $x^{ \pm}=t \pm r$, this is

$$
d s^{2}=\frac{-4 d x^{+} d x^{-}}{\left(x^{+}-x^{-}\right)^{2}}, \quad X^{2}=\frac{1-\mu x^{+} x^{-}}{x^{+}-x^{-}},
$$


with $\mu$ the mass. See e.g. [75, 92, 93] for some recent discussions on black hole formation. We recall that a 4-dim Schwarzschild black hole has a spacelike Big-Crunch singularity which is Kasner-like (3.14). One might then wonder if the Crunch (4.48) is similar to the spacelike Crunch singularity in the interior of the black hole above, where $X^{2} \rightarrow 0$ : this singular locus is

$$
1-\mu x^{+} x^{-}=0 .
$$

Here we have $x^{-}=\frac{1}{\mu x^{+}}$so the induced metric is $d s^{2}>0$ which is spacelike.

Now looking in the vicinity of the singularity, we parametrize, using $u, v$ small, as

$$
x^{+}=x_{0}^{+}+u, \quad x^{-}=x_{0}^{-}+v, \quad 1-\mu x_{0}^{+} x_{0}^{-}=0, \quad u=t+r, \quad v=t-r .
$$

Then expanding around $x_{0}^{+}=x_{0}^{-}=\frac{1}{\sqrt{\mu}}$ to linear order gives

$$
X^{2} \sim \frac{1-\mu x_{0}^{+} x_{0}^{-}-\mu\left(x_{0}^{+} v+x_{0}^{-} u\right)}{x_{0}^{+}-x_{0}^{-}+u-v} \sim \frac{-t}{r} .
$$

This is of the form of the scaling Crunch above, with $t<0$. Of course instead of looking at the interior of the black hole, we could take the scaling Crunch to be an independent cosmological solution in JT gravity, continuing the range of $t, r$ : in this case this violates standard $A d S_{2}$ boundary conditions where $X^{2} \rightarrow \frac{X_{0}^{2}}{\epsilon}$ near the boundary $r=\epsilon$. This in some ways a particular case of the general solution for the dilaton $X^{2}=\frac{1}{r}\left(a+b t+c\left(t^{2}-r^{2}\right)\right)$. It is fair to say however that any such solution can be $\mathrm{SL}(2, R)$ transformed to the black hole in the form (4.49), under appropriate conditions. So the behaviour in this case is apparently less interesting than that in the redux of the higher dimensional cases as we have seen which have more intricate structure. If we relax $A d S_{2}$ asymptotics by e.g. allowing departures from the throat region, possibly driven by extra matter, then we expect more nontrivial time-dependence to arise. It would be interesting to explore this further.

\section{Some comments on the dual field theory}

The cosmological backgrounds we have discussed with $A d S_{D}$ asymptotics $(\theta=0, z=1)$ are time-dependent deformations of AdS/CFT [24-27]: these include AdS Kasner (section 2) and other singularities. For AdS Kasner (3.19), the dual theory lives on an isotropically Crunching space $d s^{2}=-d t^{2}+t^{2 / d_{i}} d x_{i}^{2}$ with a time-dependent gauge coupling $g_{\mathrm{YM}}^{2}=e^{\Phi}=$ $t^{\alpha}$ where $\alpha>0$. Although at first sight one might imagine the dual to be weakly coupled since the gauge coupling is vanishingly small at $t \rightarrow 0$ which is the location of the bulk cosmological singularity, this turns out to not be the case and interactions are important in general. The coupling is varying rapidly since $\Phi=\alpha \log t$ so $\dot{\Phi}^{2}$ diverges. Then for instance the gauge kinetic terms $\frac{1}{g_{\mathrm{YM}}^{2}(t)} \operatorname{Tr} F^{2}$ can be transformed to canonical ones by redefining the gauge fields (as in standard perturbation theory) by absorbing the coupling into the definition of the gauge field $A_{\mu}$. However this gives rise to new tachyonic, divergent, massterms stemming from time-derivatives of the coupling. These ensure that the field variables get driven to large values as $t \rightarrow 0$. Retaining the gauge kinetic terms as above, it turns 
out that the time-dependent Schrodinger wavefunctional near $t \rightarrow 0$ has a wildly oscillating phase, and a divergent amount of energy is pumped in by the external time dependence [27]. This suggests that the gauge theory response is singular if the coupling strictly vanishes. For null time-dependence, the redefined gauge field variables (after absorbing the coupling) in fact lead to the potential mass terms vanishing due to the lightcone time dependence, thus suggesting weakly coupled Yang-Mills theory near $x^{+} \rightarrow 0$ [25]. All these arguments are subject to more detailed analysis of possible renormalization effects (with a cutoff introduced near the singularity). For instance it could be that the effective renormalized coupling does not strictly vanish, so that the dual field theory is not singular. In this case, the bulk dual might be expected to exhibit "bounce"-type behaviour. See [41, 42] for related comments on the dual to a Big-Crunch, based on a no-transmission principle.

We now describe some broader aspects of the time-dependent deformations of $A d S_{5} / C F T_{4}$ in [24-27]. These involve the bulk string theory on e.g. $A d S_{5} \times S^{5}$ (in Poincare slicing) with constant string dilaton $\Phi$, deformed as

$$
d s^{2}=\frac{R^{2}}{r^{2}}\left(\tilde{g}_{\mu \nu} d x^{\mu} d x^{\nu}+d r^{2}\right), \quad \tilde{R}_{\mu \nu}=\frac{1}{2} \partial_{\mu} \Phi \partial_{\nu} \Phi, \quad \frac{1}{\sqrt{-\tilde{g}}} \partial_{\mu}\left(\sqrt{-\tilde{g}} \tilde{g}^{\mu \nu} \partial_{\nu}\right) \Phi=0 .
$$

Here $\tilde{g}_{\mu \nu}, \Phi$ are functions of the boundary coordinates $x^{\mu}$ alone. (We are suppressing the 5 -sphere part of the metric $d \Omega_{5}^{2}$ as well as the corresponding 5-form RR-flux supporting these backgrounds.) In other words, the 4-dim "boundary" part of the bulk space is deformed but in a constrained manner, sourced by a corresponding deformation for the string dilaton $\Phi$. These constraints arise from requiring that the deformed background solves the IIB supergravity equations of motion.

With functional dependence restricted to time $t$ or null time $x^{+}$alone, we obtain timedependent deformations of $A d S / C F T$. If we deform the gauge coupling in the dual SYM to have external time-dependence as $g_{\mathrm{YM}}^{2}=e^{\Phi}=t^{\alpha}$, the rapid time variation as $t \rightarrow 0$ leads to a cosmological singularity in the bulk. Curvature singularities diverge near $t \rightarrow 0$ as e.g.

$$
R_{t t} \sim \dot{\Phi}^{2} \sim \frac{1}{t^{2}}
$$

With null time-dependence, curvature invariants are finite but tidal forces diverge, somewhat similar to [15].

The fact that these deformations are constrained has interesting consequences for the gauge theory. Since we have turned on a non-normalizable deformation for the metric, we would ordinarily expect a nonvanishing response, in particular for the stress tensor which is the dual operator. However incorporating appropriate counterterms, it turns out that the stress tensor vanishes [26]: the holographic stress tensor is

$$
T_{\mu \nu}=\frac{1}{8 \pi G_{5}}\left(K_{\mu \nu}-K h_{\mu \nu}-3 h_{\mu \nu}+\frac{1}{2} G_{\mu \nu}-\frac{1}{4} \partial_{\mu} \Phi \partial_{\nu} \Phi+\frac{1}{8} h_{\mu \nu}(\partial \Phi)^{2}\right)
$$

This is due to cancellations between distinct contributing terms arising from the metric 
deformation and the string dilaton scalar $\Phi .{ }^{1}$ This suggests that in some sense, the deformations are tuned so that the dual response vanishes. See [94] for similar effects, in the context of the Lifshitz string constructions in [60] (which are related to (5.1)). As discussed there, a smooth metric is in general expected to have various subleading coefficients nonzero in the Fefferman-Graham expansion of the metric and scalar. Conversely the deformation $g_{\mu \nu}^{(0)}$ alone will be singular, as is the case above. In the present case, it can be seen using the holographic renormalization prescriptions $[95,96]$ that in fact requiring that the subleading coefficients vanish ${ }^{2}$ leads to the constraint conditions (5.1). It is however worth noting that the above arguments are screen-dependent: changing screens using Penrose-Brown-Henneaux (PBH) transformations in fact makes the holographic stress tensor nonvanishing [26]. For instance, for AdS Kasner (3.19), the isotropically Crunching space $d s^{2}=-d t^{2}+t^{2 / d_{i}} d x_{i}^{2}$ on which the dual CFT lives is conformally flat: a PBH transformation transforms this to a new flat space screen with a nonvanishing holographic stress tensor.

Overall this suggests that the state of the gauge theory dual to the bulk theory containing cosmological singularities in the screens (5.1) is some nontrivial, exotic state: for instance severe time-dependent deformations on the vacuum state would be expected to thermalize, or equivalently lead to black hole formation in the bulk [26]. Now imagine the situation where RG effects lead to an effective renormalized coupling that does not strictly vanish: in this case the boundary theory is likely to be nonsingular, which suggests the bulk dual undergoes a nonsingular bounce. Related comments appear in [41, 42], based on a no-transmission principle. In this case, the bulk background (5.1) is likely to be deformed, thus possibly containing subleading terms in the Fefferman-Graham expansion: then the holographic stress tensor may not vanish since the cancellations in (5.3) may not occur precisely. It would be interesting to understand this more concretely.

It would appear that several of the above arguments also apply to deformations of this kind for $A d S_{4} \times X^{7}$ : in this case the scalar $\Phi$ must arise from the G-flux in Mtheory via compactification on some appropriate 7-manifold $X^{7}$. The 11-dim equations of motion would then lead to analogs of the conditions (5.1). For $\tilde{g}_{\mu \nu}$ and $\Phi$ being null deformations, this was discussed in [60]. If this also holds for time-dependent deformations,

\footnotetext{
${ }^{1}$ For a bulk metric $d s^{2}=\frac{d r^{2}}{r^{2}}+h_{\mu \nu} d x^{\mu} d x^{\nu}$ with boundary $r=$ const, the outward pointing unit normal is $n=-\frac{d r}{r}$ and the extrinsic curvature is $K_{\mu \nu}=-\frac{1}{2}\left(\nabla_{\mu} n_{\nu}+\nabla_{\nu} n_{\mu}\right)=\Gamma_{\mu \nu}^{r} n_{r}=\frac{1}{2} g^{r r}\left(-h_{\mu \nu, r}\right) n_{r}=\frac{r}{2} h_{\mu \nu, r}$. For the backgrounds (5.1), the boundary metric is $h_{\mu \nu}=\frac{1}{r^{2}} \tilde{g}_{\mu \nu}$, giving $K_{\mu \nu}=-h_{\mu \nu}$. Then the first three terms cancel: the last three terms cancel using the constraining relations between the deformations.

${ }^{2}$ Recall the Fefferman-Graham expansion for the metric $d s^{2}=\frac{d r^{2}}{r^{2}}+\frac{1}{r^{2}}\left(g_{\mu \nu}^{(0)}+r^{2} g_{\mu \nu}^{(2)}+r^{4} g_{\mu \nu}^{(4)}+\ldots\right) d x^{\mu} d x^{\nu}$ and for the scalar $\Phi=r^{(d-\Delta) / 2}\left(\Phi^{0}+r^{2} \Phi^{2}+\ldots\right)$. Then solving $R_{M N}=-4 g_{M N}+\frac{1}{2} \partial_{M} \phi \partial_{N} \phi$ iteratively gives

$$
g_{\mu \nu}^{2} \sim R_{\mu \nu}^{0}-\frac{1}{2} \partial_{\mu} \Phi \partial_{\nu} \Phi-\frac{1}{2(d-1)}\left(R-\frac{1}{2}(\partial \Phi)^{2}\right) g_{\mu \nu}^{0}: \quad g_{\mu \nu}^{2}=0 \Rightarrow R_{\mu \nu}^{0}=\frac{1}{2} \partial_{\mu} \Phi \partial_{\nu} \Phi
$$

(for a massless scalar $\Delta=d$ ) also implying the higher order coefficients vanish if $g^{(4)}=0$. Likewise, with $\square^{0}$ being the Laplacian w.r.t. $g_{\mu \nu}^{0}$, we also obtain $\Phi^{(2)} \sim \square^{0} \Phi^{0}$ : thus $\Phi^{(2)}=0$ implies $\square^{0} \Phi^{0}=0$. The backgrounds above thus appear constrained from this point of view, with only the first coefficient $g^{(0)}, \Phi^{(0)}$ nonzero for all $r$, the subleading pieces of the metric and scalar vanishing.
} 
then presumably similar comments as above will hold for the dual deformations of the ABJM theory.

In the JT case (section 4.3), the Big-Crunch scaling solution (4.48) exhibits a vanishing dilaton $X^{2} \rightarrow 0$ even at the boundary $r \rightarrow 0$, violating standard $A d S_{2}$ boundary conditions with $X^{2} \sim \frac{X_{\epsilon}^{2}}{\epsilon}$ at the boundary $r=\epsilon$. Turning on time-dependent deformations with standard $A d S_{2}$ boundary conditions is expected to lead to black hole formation (see e.g. $[75,92,93]$ which studies black hole formation in 2-dim dilaton gravity). Thus the Big-Crunch case we have discussed in JT gravity also appears to be non-generic. Perhaps correlation functions, OTOCs, string probes (see e.g. [97]) and so on will help in making more precise the signatures of the Big-Crunch here, in comparison with black holes.

The 2-dimensional backgrounds from compactifications that we have discussed are a subclass of (5.1) compatible with the KK-reduction ansatz (3.2). The analogs of the constraint conditions from the intrinsically 2 -dim point of view would appear to translate on constraints on the exponents, through the equations of motion (which are in fact what lead to the constraints (5.1)). Perhaps this is related to the universal near singular behaviour and the space of exponents being so constrained.

\section{Discussion}

We have argued for universal behaviour near Big-Bang or -Crunch singularities in various classes of theories, including flat space (3.14), AdS (4.13), (3.21), conformally AdS (4.16) and more general nonrelativistic theories with nontrivial Lifshitz scaling and hyperscaling violation (4.41). The near singularity region exhibits universal interrelations in the behaviour in time. These are essentially analogs of the familiar Kasner singularity with minimal anisotropy: embedding into various asymptotic regions gives new features however. As we have seen, it is convenient to analyse this in terms of an effective 2-dimensional dilaton-gravity-scalar theory (3.6), performing dimensional reduction as (3.2), (3.5): this then reveals the near singularity behaviour in the form (3.11), (3.13), where the dilaton potential encoding information about the asymptotic data defined by the theory has disappeared. (It is worth mentioning that the effective 2-dim analysis also helps recover various time-independent backgrounds.) This is by no means an exhaustive classification: for instance our detailed analysis based on the scaling ansatz (4.1) relied on the apparent separability of our equations of motion in time and space, leaving open the question of backgrounds that do not admit such factorization.

From the 2-dimensional point of view, the theories here are more complicated than Jackiw-Teitelboim, in particular with an extra scalar and a nontrivial dilaton potential playing essential roles in driving near singularity dynamics. We have mostly used the 2-dim theory as a crutch that faithfully captures the higher dimensional theories. Looking at the spatial profile of the dilaton $X$, we see that $X^{2} \sim r^{m}$ with $m$ reflecting a non-normalizable mode in the higher dimensional theory: for instance for the $A d S_{D}$ deformation (4.12) comprises a non-normalizable mode in $D=d_{i}+2$ dimensions, and so is distinct from one in 2-dimensions intrinsically. Likewise the spacetime metric singularities are reflected in higher dimensional curvature invariants (e.g. $R_{A B C D} R^{A B C D}$ ) diverging. However the 2- 
dim cosmological singularities are interesting in their own right: it would be interesting to understand them better, perhaps as (exotic) deformations of $A d S_{2}$, possibly using various insights from investigations of the SYK and related models and nearly $A d S_{2}$ holography e.g. [75, 86-91]. See e.g. [98] for certain classes of deformations of JT gravity.

The scaling ansatze (4.1) we have considered

$$
X^{2}=t^{k} r^{m}, \quad e^{f}=t^{a} r^{b}, \quad e^{\Phi}=t^{\alpha} r^{\beta} \rightarrow d s^{2}=\frac{e^{f}}{X^{2\left(d_{i}-1\right) / d_{i}}}\left(-d t^{2}+d r^{2}\right)+X^{4 / d_{i}} d x_{i}^{2},
$$

are designed to simulate a Big-Crunch (or -Bang), with the various fields becoming vanishingly small at some instant of time $(t=0)$ : so they are best thought of as near singularity ansatze. Through our analysis these lead to the universal interrelations $k=1, a=\frac{\alpha^{2}}{2}$, in the time-dependent behaviour, with the precise values of $\alpha$ determined by the asymptotic data. The near singular bulk region in the higher dimensional theory takes the form

$$
d s^{2}=\frac{r^{2 \theta / d_{i}}}{r^{2}}\left(\frac{1}{t^{\gamma \alpha}}\left(-\frac{d t^{2}}{r^{2 z}}+d r^{2}\right)+t^{2 / d_{i}} d x_{i}^{2}\right), \quad e^{\Phi}=t^{\alpha} r^{\beta} .
$$

For $\theta=0, z=1$, we have $\gamma=0, \beta=0$ : these are AdS Kasner singularities sourced by $\Phi$. Note that in most of the cases we have studied, the exponent $\alpha$ governing the time-dependence of $e^{\Phi}$ is irrational: this stems from the fact that $\alpha$ is determined by a quadratic (4.9). This renders tricky the possibility of analytically continuing $e^{\Phi}$ from early times $t<0$ across the singularity at $t=0$ to late times $t>0$. However an interesting exception is the $A d S_{4}$ Big-Crunch (2.3): this has $d_{i}=2, \theta=0$ so (4.9) does not give irrational $\alpha$. These are deformations of $A d S_{4} \times X^{7}$, the scalar $\Phi$ in this case arising from the 4-form flux in M-theory after compactification on some 7 -manifold $X^{7}$ : the duals are expected to be deformations of the ABJM theory.

Although the near singularity behaviour exhibits universality in terms of universal interrelations between the exponents, the precise values of the exponents are determined by the full theory. In particular this depends on the theory-dependent dilaton potential into which the singular region is embedded (and as we have seen, the space of exponents is quite constrained). The detailed form of the cosmological solution containing the BigCrunch singularity, as well as its existence, appear to depend intricately on the asymptotic region far from the singularity. This is a bit reminiscent of the UV-IR mixing discussed in [99], here in the sense that near-singularity or short-time physics intertwines with longtime physics. Note however that the analysis here is entirely classical gravity: this suggests that any such UV-IR mixing at play here is encoded within the gravity approximation. In some essential sense, the deformations are "constrained" in the sense described in section 5 , suggesting that the CFT state dual to such a cosmological singularity is some nontrivial, exotic, state, in some ways encoding information about the existence of the singularity, which perhaps reflects the UV-IR mixing above. By comparison, generic time-dependent deformations on generic CFT states would be expected to thermalize (equivalently, lead to black hole formation). It would be interesting to understand this better. In this regard, it is also worth noting that some of these geometries encode holographic flow in both space and time: e.g. the cosmological nonconformal brane backgrounds (4.16) for the D2-brane case 
$\left(d_{i}=2\right)$ might be regarded as encoding a renormalization group flow towards the M2-brane $A d S_{4}$ Kasner singularity (3.21), noting that the time-independent versions indeed encode such an RG flow encapsulated by the D2-M2 brane phase diagram [81]. In this context, see the recent paper [51]: the backgrounds there are consistent with the dimensional reduction ansatze we have discussed, and may be instructive in analysing more general flows.

It is worth noting that the scaling ansatz (6.1) above reflects a singular Big-Crunch (or -Bang) at say $t=0$ : as we have seen, this is quite constraining and leads via the detailed analysis to the precise values we have discussed. However we could relax the requirement of the fields actually Crunching fully: if instead we allow for bounce-type behaviour (with e.g. $X^{2} \stackrel{t \rightarrow 0}{\longrightarrow} X_{0}^{2} \neq 0$ and so on), then one might imagine more general possibilities. It would be interesting to explore this further.

We have retained isotropy in the spatial $x_{i}$ directions: this is consistent with the reduction to 2-dimensions. Introducing anisotropy here will of course enlarge the space of such cosmologies. It is likely that performing the kind of analysis we have done for fully anisotropic ansatze will reveal analogs of the more general anisotropic Kasner singularities embedded in various asymptotic structures: it would seem that these must exist, but it would be interesting to understand this existence question conclusively. More ambitiously it would be interesting to understand more general homogenous spaces as in the Bianchi classification to understand analogs of BKL-type singularities [100]-[104] for more general asymptotic structures (see [27] for the AdS case).

From the dual point of view, the universal near singularity bulk behaviour suggests a universal dual time-dependent strongly coupled large $N$ matrix quantum mechanics, comprising the spatial reduction of a CFT on a time-dependent base space subjected to a severe time-dependent gauge coupling. For instance the duals to the reductions to 2-dimensions of the $A d S_{5} \times S^{5}$ deformations are expected to arise from the spatial $T^{3}$ reduction of the time-dependent deformed dual $C F T_{4}$. One might imagine that this dual is some core subsector of the dual CFT possibly encoding some essential features of the bulk singularity. It appears unlikely however that this decouples from the rest of the theory: the dual is instead perhaps better thought of as the full dual theory dimensionally reduced on the compact space. We hope this point of view is of interest however in understanding the duals to cosmological singularities. In particular it would be of interest to understand possible signatures of the universal behaviour on correlation functions, OTOCs and other observables. We hope to explore this in future work.

Finally, it is interesting to recall that JT gravity was argued to be nonperturbatively dual to a random matrix model [105] (see also [106]): this gravity/ensemble duality dovetails with the existence of replica wormholes [107, 108] (see [109] for a review of some aspects of these issues). More generally this raises the question of the duals to 2-dim dilaton gravity theories being ensembles of various theories, rather than specific unitary theories (see e.g. $[98,110]$ for related discussions in more general theories). In all our discussions, we have been mostly regarding the 2-dim dilaton gravity theories as crutches for the higher dimensional theories, which we expect are dual to specific theories (see [111] for some discussions in this regard). Also, the 2-dim theories here have substantially more 
complicated dynamics than JT gravity, faithfully encoding properties of the higher dimensional theories that they are compactifications of: this suggests their behaviour is likely to be fundamentally different from that of JT gravity. It would be fascinating to understand with greater clarity if the duals to the 2-dim dilaton gravity theories here are specific theories or ensembles.

\section{Acknowledgments}

It is a pleasure to thank Daniel Grumiller for an interesting early discussion on aspects of 2-dimensional dilaton gravity from reduction, and Sumit Das, Dileep Jatkar, Arnab Kundu, Shiraz Minwalla, Sandip Trivedi and Amitabh Virmani for discussions and comments on a draft. KN also thanks Sumit Das, Shiraz Minwalla and Sandip Trivedi for many discussions over the years on AdS cosmologies and their duals. This work is partially supported by a grant to CMI from the Infosys Foundation.

Open Access. This article is distributed under the terms of the Creative Commons Attribution License (CC-BY 4.0), which permits any use, distribution and reproduction in any medium, provided the original author(s) and source are credited.

\section{References}

[1] G.T. Horowitz and A.R. Steif, Space-Time Singularities in String Theory, Phys. Rev. Lett. 64 (1990) 260 [INSPIRE].

[2] G.T. Horowitz and A.R. Steif, Strings in Strong Gravitational Fields, Phys. Rev. D 42 (1990) 1950 [INSPIRE].

[3] G. Veneziano, String cosmology: The Pre-big bang scenario, in 71st Les Houches Summer School: The Primordial Universe, Les Houches France (1999), pg. 581 [hep-th/0002094] [INSPIRE].

[4] V. Balasubramanian, S.F. Hassan, E. Keski-Vakkuri and A. Naqvi, A Space-time orbifold: A Toy model for a cosmological singularity, Phys. Rev. D 67 (2003) 026003 [hep-th/0202187] [INSPIRE].

[5] L. Cornalba and M.S. Costa, A New cosmological scenario in string theory, Phys. Rev. D 66 (2002) 066001 [hep-th/0203031] [INSPIRE].

[6] H. Liu, G.W. Moore and N. Seiberg, Strings in a time dependent orbifold, JHEP 06 (2002) 045 [hep-th/0204168] [inSPIRE].

[7] A. Lawrence, On the Instability of $3-D$ null singularities, JHEP 11 (2002) 019 [hep-th/0205288] [INSPIRE].

[8] G.T. Horowitz and J. Polchinski, Instability of space-like and null orbifold singularities, Phys. Rev. D 66 (2002) 103512 [hep-th/0206228] [InSPIRE].

[9] B. Craps, D. Kutasov and G. Rajesh, String propagation in the presence of cosmological singularities, JHEP 06 (2002) 053 [hep-th/0205101] [INSPIRE].

[10] G. Papadopoulos, J.G. Russo and A.A. Tseytlin, Solvable model of strings in a time dependent plane wave background, Class. Quant. Grav. 20 (2003) 969 [hep-th/0211289] [INSPIRE]. 
[11] K. Dasgupta, G. Rajesh, D. Robbins and S. Sethi, Time dependent warping, fluxes, and NCYM, JHEP 03 (2003) 041 [hep-th/0302049] [INSPIRE].

[12] B. Pioline and M. Berkooz, Strings in an electric field, and the Milne universe, JCAP 11 (2003) 007 [hep-th/0307280] [inSPIRE].

[13] J.L. Karczmarek and A. Strominger, Matrix cosmology, JHEP 04 (2004) 055 [hep-th/0309138] [INSPIRE].

[14] J. McGreevy and E. Silverstein, The Tachyon at the end of the universe, JHEP 08 (2005) 090 [hep-th/0506130] [INSPIRE].

[15] B. Craps, S. Sethi and E.P. Verlinde, A Matrix big bang, JHEP 10 (2005) 005 [hep-th/0506180] [INSPIRE].

[16] S.R. Das and J. Michelson, pp wave big bangs: Matrix strings and shrinking fuzzy spheres, Phys. Rev. D 72 (2005) 086005 [hep-th/0508068] [INSPIRE].

[17] B. Chen, The Time-dependent supersymmetric configurations in M-theory and matrix models, Phys. Lett. B 632 (2006) 393 [hep-th/0508191] [INSPIRE].

[18] B. Craps, A. Rajaraman and S. Sethi, Effective dynamics of the matrix big bang, Phys. Rev. D 73 (2006) 106005 [hep-th/0601062] [INSPIRE].

[19] E.J. Martinec, D. Robbins and S. Sethi, Toward the end of time, JHEP 08 (2006) 025 [hep-th/0603104] [INSPIRE].

[20] T. Ishino and N. Ohta, Matrix string description of cosmic singularities in a class of time-dependent solutions, Phys. Lett. B 638 (2006) 105 [hep-th/0603215] [INSPIRE].

[21] M. Blau and M. O'Loughlin, DLCQ and Plane Wave Matrix Big Bang Models, JHEP 09 (2008) 097 [arXiv: 0806.3255] [InSPIRE].

[22] T. Hertog and G.T. Horowitz, Towards a big crunch dual, JHEP 07 (2004) 073 [hep-th/0406134] [INSPIRE].

[23] T. Hertog and G.T. Horowitz, Holographic description of AdS cosmologies, JHEP 04 (2005) 005 [hep-th/0503071] [INSPIRE].

[24] S.R. Das, J. Michelson, K. Narayan and S.P. Trivedi, Time dependent cosmologies and their duals, Phys. Rev. D 74 (2006) 026002 [hep-th/0602107] [INSPIRE].

[25] S.R. Das, J. Michelson, K. Narayan and S.P. Trivedi, Cosmologies with Null Singularities and their Gauge Theory Duals, Phys. Rev. D 75 (2007) 026002 [hep-th/0610053] [InSPIRE].

[26] A. Awad, S.R. Das, K. Narayan and S.P. Trivedi, Gauge theory duals of cosmological backgrounds and their energy momentum tensors, Phys. Rev. D 77 (2008) 046008 [arXiv:0711.2994] [INSPIRE].

[27] A. Awad, S.R. Das, S. Nampuri, K. Narayan and S.P. Trivedi, Gauge Theories with Time Dependent Couplings and their Cosmological Duals, Phys. Rev. D 79 (2009) 046004 [arXiv:0807.1517] [INSPIRE].

[28] C.-S. Chu and P.-M. Ho, Time-dependent AdS/CFT duality and null singularity, JHEP 04 (2006) 013 [hep-th/0602054] [INSPIRE].

[29] C.-S. Chu and P.-M. Ho, Time-dependent AdS/CFT duality. II. Holographic reconstruction of bulk metric and possible resolution of singularity, JHEP 02 (2008) 058 [arXiv:0710.2640] [INSPIRE]. 
[30] F.-L. Lin and W.-Y. Wen, Supersymmetric null-like holographic cosmologies, JHEP 05 (2006) 013 [hep-th/0602124] [INSPIRE].

[31] F.-L. Lin and D. Tomino, One-loop effect of null-like cosmology's holographic dual super-Yang-Mills, JHEP 03 (2007) 118 [hep-th/0611139] [INSPIRE].

[32] N. Turok, B. Craps and T. Hertog, From big crunch to big bang with AdS/CFT, arXiv:0711.1824 [INSPIRE].

[33] B. Craps, T. Hertog and N. Turok, On the Quantum Resolution of Cosmological Singularities using AdS/CFT, Phys. Rev. D 86 (2012) 043513 [arXiv:0712.4180] [INSPIRE].

[34] B. Craps, F. De Roo and O. Evnin, Can free strings propagate across plane wave singularities?, JHEP 03 (2009) 105 [arXiv:0812.2900] [INSPIRE].

[35] K. Madhu and K. Narayan, String spectra near some null cosmological singularities, Phys. Rev. D 79 (2009) 126009 [arXiv:0904.4532] [InSPIRE].

[36] A. Awad, S.R. Das, A. Ghosh, J.-H. Oh and S.P. Trivedi, Slowly Varying Dilaton Cosmologies and their Field Theory Duals, Phys. Rev. D 80 (2009) 126011 [arXiv:0906.3275] [INSPIRE].

[37] E.J. Copeland, G. Niz and N. Turok, The string wave function across a Kasner singularity, Phys. Rev. D 81 (2010) 126006 [arXiv:1001.5291] [INSPIRE].

[38] X. Dong, B. Horn, E. Silverstein and G. Torroba, Unitarity bounds and RG flows in time dependent quantum field theory, Phys. Rev. D 86 (2012) 025013 [arXiv:1203.1680] [INSPIRE].

[39] N. Engelhardt, T. Hertog and G.T. Horowitz, Holographic Signatures of Cosmological Singularities, Phys. Rev. Lett. 113 (2014) 121602 [arXiv:1404.2309] [INSPIRE].

[40] N. Engelhardt, T. Hertog and G.T. Horowitz, Further Holographic Investigations of Big Bang Singularities, JHEP 07 (2015) 044 [arXiv: 1503.08838] [INSPIRE].

[41] N. Engelhardt and G.T. Horowitz, Holographic Consequences of a No Transmission Principle, Phys. Rev. D 93 (2016) 026005 [arXiv:1509.07509] [INSPIRE].

[42] N. Engelhardt and G.T. Horowitz, New Insights into Quantum Gravity from Gauge/gravity Duality, Int. J. Mod. Phys. D 25 (2016) 1643002 [arXiv:1605.04335] [InSPIRE].

[43] S. Banerjee, S. Bhowmick, S. Chatterjee and S. Mukherji, A note on AdS cosmology and gauge theory correlator, JHEP 06 (2015) 043 [arXiv:1501.06317] [INSPIRE].

[44] J.L.F. Barbón and E. Rabinovici, Holographic complexity and spacetime singularities, JHEP 01 (2016) 084 [arXiv: 1509.09291] [INSPIRE].

[45] S.P. Kumar and V. Vaganov, Probing crunching AdS cosmologies, JHEP 02 (2016) 026 [arXiv: 1510.03281] [INSPIRE].

[46] R.H. Brandenberger, E.G.M. Ferreira, I.A. Morrison, Y.-F. Cai, S.R. Das and Y. Wang, Fluctuations in a cosmology with a spacelike singularity and their gauge theory dual description, Phys. Rev. D 94 (2016) 083508 [arXiv:1601.00231] [InSPIRE].

[47] J. Choi, J.J. Fernandez-Melgarejo and S. Sugimoto, Supersymmetric Gauge Theory with Space-time-Dependent Couplings, PTEP 2018 (2018) 013B01 [arXiv:1710.09792] [INSPIRE].

[48] H. Bernardo and H. Nastase, Holographic cosmology from "dimensional reduction" of $\mathcal{N}=4$ $S Y M$ vs. $A d S_{5} \times S^{5}$, JHEP 12 (2019) 025 [arXiv:1812.07586] [INSPIRE]. 
[49] S.R. Das, S. Hampton and S. Liu, Quantum quench in $c=1$ matrix model and emergent space-times, JHEP 04 (2020) 107 [arXiv:1910.00123] [INSPIRE].

[50] A. Arduino, R. Finotello and I. Pesando, On the origin of divergences in time-dependent orbifolds, Eur. Phys. J. C 80 (2020) 476 [arXiv:2002.11306] [INSPIRE].

[51] A. Frenkel, S.A. Hartnoll, J. Kruthoff and Z.D. Shi, Holographic flows from CFT to the Kasner universe, arXiv:2004.01192 [INSPIRE].

[52] D.F.Z. Marchioro and D.L. Nedel, Left/right entanglement and thermalization of time dependent plane wave Green-Schwarz superstring, JHEP 07 (2020) 102 [arXiv:2005.09040] [INSPIRE].

[53] S. Choudhury, The Cosmological OTOC: Formulating new cosmological micro-canonical correlation functions for random chaotic fluctuations in Out-of-Equilibrium Quantum Statistical Field Theory, arXiv:2005.11750 [INSPIRE].

[54] B. Craps, Big Bang Models in String Theory, Class. Quant. Grav. 23 (2006) S849 [hep-th/0605199] [INSPIRE].

[55] C.P. Burgess and L. McAllister, Challenges for String Cosmology, Class. Quant. Grav. 28 (2011) 204002 [arXiv: 1108.2660] [INSPIRE].

[56] J.M. Maldacena, The Large $N$ limit of superconformal field theories and supergravity, Int. $J$. Theor. Phys. 38 (1999) 1113 [hep-th/9711200] [INSPIRE].

[57] S.S. Gubser, I.R. Klebanov and A.M. Polyakov, Gauge theory correlators from noncritical string theory, Phys. Lett. B 428 (1998) 105 [hep-th/9802109] [INSPIRE].

[58] E. Witten, Anti-de Sitter space and holography, Adv. Theor. Math. Phys. 2 (1998) 253 [hep-th/9802150] [INSPIRE].

[59] O. Aharony, S.S. Gubser, J.M. Maldacena, H. Ooguri and Y. Oz, Large N field theories, string theory and gravity, Phys. Rept. 323 (2000) 183 [hep-th/9905111] [INSPIRE].

[60] K. Balasubramanian and K. Narayan, Lifshitz spacetimes from AdS null and cosmological solutions, JHEP 08 (2010) 014 [arXiv: 1005.3291] [INSPIRE].

[61] A. Strominger, Les Houches lectures on black holes, in NATO Advanced Study Institute: Les Houches Summer School, Session 62: Fluctuating Geometries in Statistical Mechanics and Field Theory, Les Houches France (1994) [hep-th/9501071] [INSPIRE].

[62] S. Nojiri and S.D. Odintsov, Quantum dilatonic gravity in $(D=2)$-dimensions, $(D=4)$-dimensions and $(D=5)$-dimensions, Int. J. Mod. Phys. A 16 (2001) 1015 [hep-th/0009202] [INSPIRE].

[63] D. Grumiller, Quantum dilaton gravity in two-dimensions with matter, gr-qc/0105078 [INSPIRE].

[64] D. Grumiller, W. Kummer and D.V. Vassilevich, Dilaton gravity in two-dimensions, Phys. Rept. 369 (2002) 327 [hep-th/0204253] [INSPIRE].

[65] R.B. Mann and S.F. Ross, Gravitation and cosmology in $(1+1)$-dimensional dilaton gravity, Phys. Rev. D 47 (1993) 3312 [hep-th/9206022] [inSPIRE].

[66] F.D. Mazzitelli and J.G. Russo, Dilaton quantum cosmology in two-dimensions, Phys. Rev. D 47 (1993) 4490 [hep-th/9211095] [INSPIRE].

[67] R. Moessner and M. Trodden, Singularity - free two-dimensional cosmologies, Phys. Rev. D 51 (1995) 2801 [gr-qc/9405004] [INSPIRE]. 
[68] O.B. Zaslavskii, Exactly solvable models of two-dimensional dilaton cosmology with quantum back reaction, Class. Quant. Grav. 20 (2003) 2963 [hep-th/0305199] [INSPIRE].

[69] M.B. Christmann, F.P. Devecchi, G.M. Kremer and C.M. Zanetti, Transition from accelerated to decelerated regimes in JT and CGHS cosmologies, Europhys. Lett. 67 (2004) 728 [gr-qc/0407029] [INSPIRE].

[70] L.L. Samojeden, G.M. Kremer and F.P. Devecchi, Accelerated expansion in bosonic and fermionic $2 D$ cosmologies with quantum effects, Europhys. Lett. 87 (2009) 10001 [arXiv:0906.3315] [INSPIRE].

[71] J. Govaerts and S. Zonetti, Quantized cosmological constant in $1+1$ dimensional quantum gravity with coupled scalar matter, Class. Quant. Grav. 28 (2011) 185001 [arXiv:1102.4957] [INSPIRE].

[72] J.P.M. Pitelli, Quantum Cosmology in $(1+1)$-dimensional Hor̆ava-Lifshitz theory of gravity, Phys. Rev. D 93 (2016) 104024 [arXiv: 1605.01979] [INSPIRE].

[73] S. Nojiri and S.D. Odintsov, Novel cosmological and black hole solutions in Einstein and higher-derivative gravity in two dimensions, Europhys. Lett. 130 (2020) 10004 [arXiv: 2004.01404] [INSPIRE].

[74] C.G. Callan Jr., S.B. Giddings, J.A. Harvey and A. Strominger, Evanescent black holes, Phys. Rev. D 45 (1992) 1005 [hep-th/9111056] [INSPIRE].

[75] A. Almheiri and J. Polchinski, Models of AdS 2 backreaction and holography, JHEP 11 (2015) 014 [arXiv: 1402.6334] [INSPIRE].

[76] K.S. Kolekar and K. Narayan, AdS 2 dilaton gravity from reductions of some nonrelativistic theories, Phys. Rev. D 98 (2018) 046012 [arXiv:1803.06827] [INSPIRE].

[77] K.S. Kolekar and K. Narayan, On $A d S_{2}$ holography from redux, renormalization group flows and c-functions, JHEP 02 (2019) 039 [arXiv: 1810.12528] [INSPIRE].

[78] D. Grumiller, D. Hofmann and W. Kummer, Two dilaton theories in two-dimensions, Annals Phys. 290 (2001) 69 [gr-qc/0005098] [INSPIRE].

[79] M. Taylor, Lifshitz holography, Class. Quant. Grav. 33 (2016) 033001 [arXiv:1512.03554] [INSPIRE].

[80] S.A. Hartnoll, A. Lucas and S. Sachdev, Holographic quantum matter, arXiv:1612.07324 [INSPIRE].

[81] N. Itzhaki, J.M. Maldacena, J. Sonnenschein and S. Yankielowicz, Supergravity and the large $N$ limit of theories with sixteen supercharges, Phys. Rev. D 58 (1998) 046004 [hep-th/9802042] [INSPIRE].

[82] X. Dong, S. Harrison, S. Kachru, G. Torroba and H. Wang, Aspects of holography for theories with hyperscaling violation, JHEP 06 (2012) 041 [arXiv:1201.1905] [INSPIRE].

[83] D. Mukherjee and K. Narayan, Hyperscaling violation, quasinormal modes and shear diffusion, JHEP 12 (2017) 023 [arXiv:1707.07490] [INSPIRE].

[84] R. Jackiw, Lower Dimensional Gravity, Nucl. Phys. B 252 (1985) 343 [InSPIRE].

[85] C. Teitelboim, Gravitation and Hamiltonian Structure in Two Space-Time Dimensions, Phys. Lett. B 126 (1983) 41 [inSPIRE]. 
[86] J. Maldacena, D. Stanford and Z. Yang, Conformal symmetry and its breaking in two dimensional Nearly Anti-de-Sitter space, PTEP 2016 (2016) 12C104 [arXiv:1606.01857] [INSPIRE].

[87] K. Jensen, Chaos in AdS 2 Holography, Phys. Rev. Lett. 117 (2016) 111601 [arXiv: 1605.06098] [INSPIRE].

[88] J. Engelsöy, T.G. Mertens and H. Verlinde, An investigation of $A d S_{2}$ backreaction and holography, JHEP 07 (2016) 139 [arXiv:1606.03438] [INSPIRE].

[89] G. Sárosi, $A d S_{2}$ holography and the $S Y K$ model, PoS (Modave2017) 001 [arXiv:1711.08482] [INSPIRE].

[90] V. Rosenhaus, An introduction to the SYK model, J. Phys. A 52 (2019) 323001 [arXiv: 1807.03334] [INSPIRE].

[91] D.A. Trunin, Pedagogical introduction to SYK model and 2D Dilaton Gravity, arXiv:2002.12187 [INSPIRE].

[92] A. Dhar, A. Gaikwad, L.K. Joshi, G. Mandal and S.R. Wadia, Gravitational collapse in SYK models and Choptuik-like phenomenon, JHEP 11 (2019) 067 [arXiv:1812.03979] [INSPIRE].

[93] U. Moitra, S.K. Sake, S.P. Trivedi and V. Vishal, Jackiw-Teitelboim Model Coupled to Conformal Matter in the Semi-Classical Limit, JHEP 04 (2020) 199 [arXiv:1908.08523] [INSPIRE].

[94] K. Narayan, AdS null deformations with inhomogeneities, Phys. Rev. D 86 (2012) 126004 [arXiv: 1209.4348] [INSPIRE].

[95] S. de Haro, S.N. Solodukhin and K. Skenderis, Holographic reconstruction of spacetime and renormalization in the AdS/CFT correspondence, Commun. Math. Phys. 217 (2001) 595 [hep-th/0002230] [INSPIRE].

[96] K. Skenderis, Lecture notes on holographic renormalization, Class. Quant. Grav. 19 (2002) 5849 [hep-th/0209067] [INSPIRE].

[97] A. Banerjee, A. Kundu and R. Poojary, Maximal Chaos from Strings, Branes and Schwarzian Action, JHEP 06 (2019) 076 [arXiv:1811.04977] [INSPIRE].

[98] E. Witten, Deformations of JT Gravity and Phase Transitions, arXiv:2006.03494 [INSPIRE].

[99] S. Minwalla, M. Van Raamsdonk and N. Seiberg, Noncommutative perturbative dynamics, JHEP 02 (2000) 020 [hep-th/9912072] [INSPIRE].

[100] L.D. Landau and E.M. Lifshitz, Course of Theoretical Physics. Vol. 2: Classical Theory of Fields, Pergamon, Oxford U.K. (1987).

[101] E. Lifshitz, V. Belinskii and I. Khalatnikov, Oscillatory approach to a singular point in the relativistic cosmology, Adv. Phys. 19 (1970) 525.

[102] V.A. Belinski and I.M. Khalatnikov, Effect of Scalar and Vector Fields on the Nature of the Cosmological Singularity, Sov. Phys. JETP 36 (1973) 591 [inSPIRE].

[103] C.W. Misner, Quantum cosmology. 1., Phys. Rev. 186 (1969) 1319 [inSPIRE].

[104] T. Damour, M. Henneaux and H. Nicolai, Cosmological billiards, Class. Quant. Grav. 20 (2003) R145 [hep-th/0212256] [INSPIRE]. 
[105] P. Saad, S.H. Shenker and D. Stanford, JT gravity as a matrix integral, arXiv:1903.11115 [INSPIRE].

[106] D. Stanford and E. Witten, JT Gravity and the Ensembles of Random Matrix Theory, arXiv: 1907.03363 [INSPIRE].

[107] G. Penington, S.H. Shenker, D. Stanford and Z. Yang, Replica wormholes and the black hole interior, arXiv: 1911.11977 [INSPIRE].

[108] A. Almheiri, T. Hartman, J. Maldacena, E. Shaghoulian and A. Tajdini, Replica Wormholes and the Entropy of Hawking Radiation, JHEP 05 (2020) 013 [arXiv:1911.12333] [INSPIRE].

[109] A. Almheiri, T. Hartman, J. Maldacena, E. Shaghoulian and A. Tajdini, The entropy of Hawking radiation, arXiv:2006.06872 [INSPIRE].

[110] D. Marolf and H. Maxfield, Transcending the ensemble: baby universes, spacetime wormholes, and the order and disorder of black hole information, arXiv:2002.08950 [INSPIRE].

[111] J. McNamara and C. Vafa, Baby Universes, Holography, and the Swampland, arXiv: 2004.06738 [INSPIRE]. 